\title{
Impact of Hyperbaric Oxygen Therapy on Cognitive Functions: a Systematic Review
}

\author{
Anna B. Marcinkowska ${ }^{1,2}$ (]) Natalia D. Mankowska ${ }^{1}$. Jacek Kot ${ }^{3} \cdot$ Pawel J. Winklewski $^{1,2}$
}

Received: 17 May 2020 / Accepted: 7 March 2021 / Published online: 13 April 2021

(c) The Author(s) 2021

\begin{abstract}
Hyperbaric oxygen therapy (HBOT) is a modality of treatment in which patients inhale $100 \%$ oxygen inside a hyperbaric chamber pressurised to greater than 1 atmosphere. The aim of this review is to discuss neuropsychological findings in various neurological disorders treated with HBOT and to open new perspectives for therapeutic improvement. A literature search was conducted in the MEDLINE (via PubMed) database from the inception up 10 May 2020. Eligibility criteria included original articles published in English. Case studies were excluded. Full-text articles were obtained from the selected studies and were reviewed on the following inclusion criteria (1) performed cognitive processes assessment (2) performed HBOT with described protocol. Two neuropsychologists independently reviewed titles, abstracts, full texts and extracted data. The initial search retrieved 1024 articles, and a total of 42 studies were finally included after applying inclusion and exclusion criteria. The search yielded controversial results with regard to the efficiency of HBOT in various neurological conditions with cognitive disturbance outcome. To the best of our knowledge this is the first state-of-the art, systematic review in the field. More objective and precise neuropsychological assessment methods are needed to exact evaluation of the efficacy of HBOT for neuropsychological deficits. Future studies should widen the assessment of HBOT effects on different cognitive domains because most of the existing studies have focussed on a single process. Finally, there is a need for further longitudinal studies.
\end{abstract}

Keywords Neuropsychology $\cdot$ Cognition $\cdot$ Hyperbaric oxygen therapy

\section{Key Points}

- Enhanced oxygen supply and increased pressure result in a variety of pathophysiological mechanisms.

- A more aggressive approach to carbon monoxide poisoning is needed to ameliorate cognitive consequences.

- A post-concussion syndrome trials should include standardised neuropsychological methods.

- Hyperbaric oxygen therapy could be considered in stroke patients after thrombolysis or thrombectomy.

- Hyperbaric oxygen therapy for treating dementia should be further investigated.

Anna B. Marcinkowska anna.marcinkowska@gumed.edu.pl

1 Applied Cognitive Neuroscience Lab, Department of Human Physiology, Medical University of Gdańsk, Tuwima Str. 15 80-210, Gdańsk, Poland

2 2nd Department of Radiology, Medical University of Gdańsk, Gdańsk, Poland

3 National Centre for Hyperbaric Medicine, Institute of Maritime and Tropical Medicine in Gdynia, Medical University of Gdansk, Gdańsk, Poland

\section{Introduction}

Hyperbaric oxygen therapy (HBOT) is a modality of treatment in which patients inhale $100 \%$ oxygen through a head tent, mask or endotracheal tube inside a hyperbaric chamber that has been pressurised to greater than 1 absolute atmosphere (ATA). HBOT is typically administered at more than one and less than three ATA and induces a state of increased pressure and hyperoxia that cause mechanical and physiologic effects.

HBOT has been recommended for various conditions for more than 40 years (Grim, 1990; Leach et al., 1998). Initially, it was used to treat decompression sickness in divers. However, over the years its far-reaching potential has been recognised, and it has been approved for a variety of purposes, including carbon monoxide (CO) poisoning, decompression sickness and gas embolism, problematic wound healing, delayed radiation injuries, sudden deafness and other conditions as indicated by evidence-based medicine (Mathieu et al., 2017; Moon, 2019). Although controlled clinical trials are limited, the rational basis of HBOT as well 
as good clinical results have gradually increased the use of HBOT for neurological disorders linked with cognitive disturbances. Neurological disorders as well as conditions related to central nervous system (CNS) damage may present with a variety of neuropsychological symptoms, such as impairment of memory and learning processes, attention and visuo-spatial functions, language processes and executive dysfunctions.

The neuroprotective and therapeutic effects of neuropsychological deficits provided by HBOT have been established in experimental animal and human models, although they remain controversial. In this review, we will summarise the existing results of HBOT usage in several neurological states, its current understanding and opinions for future studies. To the best of our knowledge this is the first state of the art, systematic review in this topic.

To understand the role of HBOT in neurological disorders, a basic knowledge of cerebral metabolism, cerebral blood flow and the neurophysiology of the brain is essential. The physiological basis of HBOT is the gas laws. While breathing air at atmospheric pressure, most of the oxygen is bound to haemoglobin. In this situation, blood haemoglobin is saturated with oxygen (at approximately 97\%) and working at near maximum capacity with a small amount of oxygen dissolved in the blood plasma compartment. If the percent of inhaled oxygen - or the pressure at which oxygen is breathed - is increased, more oxygen will dissolve into the blood plasma. In HBOT conditions, the fraction of inspired oxygen and partial pressure of oxygen increases, supersaturating the blood with oxygen. Inhaling 100\% oxygen at 3 ATA increases mean arterial oxygen tension from approximately $100 \mathrm{mmHg}$ in normobaric conditions to $2000 \mathrm{mmHg}$ and the amount of oxygen delivery to the tissues from 3 to $60 \mathrm{~mL}$ of oxygen per litre of blood (Jain, 2017). Supersaturation of the blood to this degree supports resting tissue oxygen requirements without a necessary contribution from haemoglobin carriage. Hence, HBOT is useful in diseases in which haemoglobin function is limited, such as $\mathrm{CO}$ poisoning or ischaemia (Mathieu, 2006a). The excess oxygen is carried in solution and it can diffuse to areas where red blood cells cannot reach. This elevation in the partial pressure of oxygen in tissue mediates the therapeutic benefits of HBOT.

Enhanced oxygen supply and increased pressure result in a wide variety of pathophysiological mechanisms. Thus, HBOT is believed to diminish neuroinflammatory responses, blood-brain barrier permeability and apoptosis while positively impacting neurogenesis, neuronal and axonal integrity and synaptogenesis (reviewed by Daly et al., 2018; Fischer et al., 2010). All of these effects may potentially influence patients' cognitive functioning. Nevertheless, direct links are very difficult to establish. Recent advances in radiology and medical imaging, in particular diffusion tensor imaging (DTI) and advanced perfusion models, could potentially fill the gap and provide better understanding of the interdependence among white matter structure, cerebral blood flow and cognition (Chen et al., 2010; Tal et al., 2017).

Brain metabolism generates large amounts of reactive forms of oxygen. There is an excessive literature demonstrating that augmented oxygen reactivity is associated with deteriorated performance in cognitive tasks (Bhatt et al., 2020; Kandlur et al., 2020). At the same time, however, reactive oxygen species may also play a role in functional and structural modifications indispensable for synaptic plasticity (Massaad \& Klann, 2011). In particular reactive forms of oxygen seem to be involved in regulation of N-methyl-d-aspartate (NMDA) receptors (Betzen et al., 2009), calcium $\left(\mathrm{Ca}^{2+}\right)$ channels (Huddleston et al., 2008), potassium channels (Gong et al., 2000) and $\mathrm{Ca}^{2+} /$ calmodulin kinase II (Shetty et al., 2008) functioning. Hydrogen peroxide promotes ryanodine receptor redox modifications in hippocampal neurons (Kemmerling et al., 2007). Consequently, although oxygen reactive forms seem to be necessary for long term potentiation (the cellular substrate for memory), they are also implicated with deficient long term potentiation during aging (Hu et al., 2007) and in mouse models of Alzheimer Disease (AD) (Cai et al., 2008). HBOT schemes differ from study to study what may result in various exposures to oxidative stress. Therefore, it is difficult to predict whether beneficial or detrimental effects of reactive oxygen forms on cognition in particular study prevails.

The diversity and strength of innate repair mechanisms activated by HBOT are associated both with the elevated level of dissolved oxygen and the elevated pressure (Efrati \& Ben-Jacob, 2014b). However, it remains unclear how oxygen and pressure relate to each other. For instance, it has been proposed that HBOT may act as a transducer to improve oxidative metabolism while subsequent normobaric oxygen therapy (100\% oxygen, 1 ATA) may further enhance this effect (Rockswold et al., 2010, 2013). Exposure of rat brain slices to high pressures (5.3 ATA) typically shows an augmentation in the synaptic NMDA receptors response, usually followed by post-synaptic excitability modifications (Bliznyuk et al., 2020; Mor \& Grossman, 2010; Mor \& Grossman, 2007). HBOT and normobaric reoxygenation augment excitability and activate oxygen-induced potentiation in CA1 hippocampal neurons (Garcia et al., 2010b). Moreover, oxygen induced neural plasticity does not need modifications in excitatory synaptic transmission (Garcia et al., 2010a). When referring to experiments performed with the use of brain slices it should be kept in mind that it remains unclear whether the reported hyperexcitability, and which forms of increased neuronal excitability are a part of the learning process or, actually represent cellular manifestation of neuronal oxygen poisoning. The only experiments (performed by our team) on humans exposed to 1.4 
ATA and 2.8 ATA HBO may suggest that actually oxygen related mechanisms affecting cognition and poisoning might be interrelated (Kot et al., 2015). The study was, however, performed on young males from the elite military Special Forces unit and therefore might not be representative for wider population.

Traditional view is that hyperoxia (below 2.8 ATA) diminishes brain perfusion (Di Piero et al., 2002; Kety \& Schmidt, 1948; Omae et al., 1998) due to cerebral vasoconstriction caused by an interruption in nitric oxide-mediated basal relaxation of cerebral vessels (Bitterman \& Bitterman, 1998; Oury et al., 1992). The topic has been extensively reviewed by our team (Winklewski et al., 2013). The existing dogma has been challenged by Micarelli et al. (2013). Authors investigated 7 healthy volunteers during acute exposure to HBO at 2.5 ATA (thus mimicking a possible therapeutic condition). The authors reported augmented brain perfusion in the left hemisphere with a relative cerebral blood flow increase in the neural networks encompassing dorsal and ventral attention pathways. Nevertheless, the links between changes in brain perfusion and cognition are complex and not fully understood. Quite insightful are data coming from the investigations of the impact of physical training on perfusion and cognition. It is generally believed that active lifestyle slowdown cerebral prefusion and cognitive decline associated with age (Boraxbekk et al., 2016; Jackson et al., 2016; Szalewska et al., 2017). However, Guiney et al. (2019) demonstrated that improvements in higher-order cognitive functioning in healthy older adults associated with habitual physical activity were not related to improved cerebrovascular functioning.

Hyperoxia and in particular HBOT exposure results in increased parasympathetic nervous system activity, bradycardia and dissociation between heart rate and broader cardiovascular system responses (Lodato \& Jubran, 1993; Parkinson \& Registrar, 1871; Walter et al., 1962). The neurovisceral integration model proposes a bi-directional cortical influences on autonomic functioning and integrates central nervous and autonomic systems (Smith et al., 2017; Thayer \& Lane, 2000). The main affector and effector of the parasympathetic nervous system is the vagal nerve. Electrical vagal nerve stimulation seems to improve cognitive functioning, in particular memory consolidation and recognition (Clark et al., 1999; Ghacibeh et al., 2006; Vonck et al., 2014). Interestingly, also behavioural interventions such as slow breathing, associated with vagal nerve increased activity, are able to modulate cortical alpha rhythm (Hsu et al., 2020; Maric et al., 2020). Cardiovascular desynchronization, in turn, may have detrimental impact on cognition (Ogoh \& Tarumi, 2019).

Evidence for cognitive improvements after HBOT in different neurological disorders accumulates (Boussi-Gross et al., 2015; Hardy et al., 2002; Rossignol et al., 2007;
Tapeantong \& Poungvarin, 2009). Nevertheless, there is still an ongoing debate regarding the efficacy of HBOT in individuals with traumatic brain injury (TBI), stroke or other neurological conditions. Although it is widely agreed, and confirmed by the majority of studies, that HBOT leads to significant improvements in cognition, the debate is mostly related to a control group issue and minimal effective dosages (the minimal pressure that does not have any physiological effect on the CNS) (Churchill et al., 2013; Efrati \& Ben-Jacob, 2014a, b; Golden et al., 2006; Harch et al., 2007; Wolf et al., 2012). There are several neurological conditions where HBOT has been reported to be useful, that is, $\mathrm{CO}$ poisoning, TBI and post-concussion syndrome (PCS), stroke and neurodegenerative disorders.

Existing intensive functional therapy and neuropsychological rehabilitation programmes are considered essential to minimise cognitive and physical sequelae associated with brain damage in various neurological states. Neuropsychological rehabilitation refers to helping cognitively impaired individuals to totally or partially restore normal functioning, compensate cognitive, emotional, psychosocial and behavioural deficits, and improve the quality of life. However, considering the heterogeneity of physical, cognitive, behavioural, and psychosocial symptoms in various neurological states, these programmes are often only partially successful. Those programs should be improved and modified in accordance with the better understanding of cognitive processes and brain structures via newest neuroimaging techniques and medical development. It is well known that neuroplasticity and synaptic reorganization have a decisive role in neuropsychological rehabilitation effects (Sohlberg \& Mateer, 2001). Some of the mechanisms involved in both processes have essential implications for rehabilitation, such as diaschisis, functional reorganization, or modification of synaptic connectivity among others (Sohlberg \& Mateer, 2001). Thus, alternative approaches dedicated to the metabolic recovery of cerebral tissues that stimulates synaptic and functional plasticity such as HBOT need to be further explored in order to improve the neuropsychological outcome of a patient.

Finally, there are some adverse events and risks associated with HBOT. They are related to changes in atmospheric pressure, hyperbaric oxygen exposure and psychological factors (i.e. confinement anxiety). Middle ear barotrauma is the most common HBOT-related complication with incidence rates ranging between authors from 2 to $82 \%$. In one of the largest retrospective study of HBOT safety the incident risk of middle era barotrauma was $9.2 \%$ of patients, which gives 410 events per 100,000 sessions. The safety of hyperbaric oxygen treatment was assessed in 2,334 patients (Hadanny et al., 2016b). To minimise this risk, patients are provided with instructions describing ear clearing techniques like swallowing, chewing and modified Valsalva manoeuvre. In intubated and unconscious patients 
myringotomy is the procedure of choice before the HBOT. Oxygen itself is a drug and there is always a risk of adverse effects based on biochemical reactions. CNS oxygen toxicity presented with the temporarily loss of consciousness and general seizures is quite infrequent event. It occurs with a rate of only 1.59 events per 100,000 sessions, mostly at pressures 2.4 ATA and above (Hadanny et al., 2016a). Seizures during hyperbaric oxygen therapy were assessed in retrospective analysis of 62,614 treatment sessions. This is one of the reasons why in case of using HBOT in emergency indications (i.e. diving accidents, $\mathrm{CO}$ intoxication, compartment syndrome, etc.) when high partial pressure of oxygen is used, use of multiplace chambers with direct supervision of medical attendant inside the chamber is preferred. If such event occurs, the internal attendant immediately removes the oxygen mask from the patient and switches him or her to air breathing. Lungs oxygen toxicity is cumulative, but can be avoided by using minimum inspired fractions of oxygen between HBOT sessions. In some patients with pre-existing cardiac problems, there is a risk of hypotension after HBOT due to hypovolemia evoked by vanishing vasoconstrictive effect. Hypovolemia risk can be mitigated by careful volume assessment and, if needed, volume loading before the end of oxygen exposure. Eyes oxygen toxicity, manifesting as myopia, may occur in patients receiving HBOT over several weeks. Myopia is usually spontaneously reversible within a short period of time. Gas embolism and pulmonary barotrauma, a well-known phenomena in divers, during decompression in hyperbaric chambers are very rare (Mathieu, 2006b; Oriani et al., 1996). In conclusion of the adverse effects, the HBOT is generally considered as a low risk medical procedure when conducted by the well trained hyperbaric personnel in carefully assessed patients.

\section{Methodology}

PRISMA guidelines were used for the reporting of this systematic review (Moher et al., 2009).

\section{Data Sources}

Electronic database Pubmed was searched, from 1946 to 2020 , for relevant studies; last search was performed on 10 May 2020. The reference lists of included studies were handsearched for additional references.

Separate search for four medical indications for HBOT (CO intoxication, post-concussion syndrome, stroke, ageing and neurodegenerative diseases) was made. The following search terms were used: a) CO intoxication Search: ((hyperbaric oxygen therapy) AND (carbon monoxide)) AND (cognition) ("hyperbaric oxygenation"[MeSH Terms] OR ("hyperbaric"[All Fields] AND "oxygenation"[All Fields]) OR "hyperbaric oxygenation"[All Fields] OR ("hyperbaric"[All Fields] AND "oxygen"[All Fields] AND "therapy"[All Fields]) OR "hyperbaric oxygen therapy"[All Fields]) AND ("carbon monoxide"[MeSH Terms] OR ("carbon"[All Fields] AND "monoxide"[All Fields]) OR "carbon monoxide"[All Fields]) AND ("cognition"[MeSH Terms] OR "cognition"[All Fields] OR "cognitions"[All Fields] OR "cognitive"[All Fields] OR "cognitively"[All Fields] OR "cognitives"[All Fields])

b) Traumatic brain injury Search: ((hyperbaric oxygen therapy) AND (concussion)) AND (cognition) Sort by: Most Recent ("hyperbaric oxygenation"[MeSH Terms] OR ("hyperbaric"[All Fields] AND "oxygenation"[All Fields]) OR "hyperbaric oxygenation"[All Fields] OR ("hyperbaric"[All Fields] AND "oxygen"[All Fields] AND "therapy"[All Fields]) OR "hyperbaric oxygen therapy"[All Fields]) AND ("brain concussion"[MeSH Terms] OR ("brain"[All Fields] AND "concussion"[All Fields]) OR "brain concussion"[All Fields] OR "concussion"[All Fields] OR "concussions"[All Fields] OR "concussed"[All Fields] OR "concussive"[All Fields]) AND ("cognition"[MeSH Terms] OR "cognition"[All Fields] OR "cognitions"[All Fields] OR "cognitive"[All Fields] OR "cognitively"[All Fields] OR "cognitives"[All Fields]) and Search: ((hyperbaric oxygen therapy) AND (traumatic brain injury)) AND (cognition) ("hyperbaric oxygenation"[MeSH Terms] OR ("hyperbaric"[All Fields] AND "oxygenation"[All Fields]) OR "hyperbaric oxygenation"[All Fields] OR ("hyperbaric"[All Fields] AND "oxygen"[All Fields] AND "therapy"[All Fields]) OR "hyperbaric oxygen therapy"[All Fields]) AND ("brain injuries, traumatic"[MeSH Terms] OR ("brain"[All Fields] AND "injuries"[All Fields] AND "traumatic"[All Fields]) OR "traumatic brain injuries"[All Fields] OR ("traumatic"[All Fields] AND "brain"[All Fields] AND "injury"[All Fields]) OR "traumatic brain injury"[All Fields]) AND ("cognition"[MeSH Terms] OR "cognition"[All Fields] OR "cognitions"[All Fields] OR "cognitive"[All Fields] OR "cognitively"[All Fields] OR "cognitives"[All Fields])

c) Stroke Search: ((hyperbaric oxygen therapy) AND (stroke)) AND (cognition) ("hyperbaric oxygenation"[MeSH Terms] OR ("hyperbaric"[All Fields] AND "oxygenation"[All Fields]) OR "hyperbaric oxygenation"[All Fields] OR ("hyperbaric"[All Fields] AND "oxygen"[All Fields] AND "therapy"[All Fields]) OR "hyperbaric oxygen therapy"[All Fields]) AND 
("stroke"[MeSH Terms] OR "stroke"[All Fields] OR "strokes"[All Fields] OR "stroke s"[All Fields]) AND ("cognition"[MeSH Terms] OR "cognition"[All Fields] OR "cognitions"[All Fields] OR "cognitive"[All Fields] OR "cognitively"[All Fields] OR "cognitives"[All Fields]) and Search: ((hyperbaric oxygen therapy) AND (cognition)) AND (ischemia) ("hyperbaric oxygenation"[MeSH Terms] OR ("hyperbaric"[All Fields] AND "oxygenation"[All Fields]) OR "hyperbaric oxygenation"[All Fields] OR ("hyperbaric"[All Fields] AND "oxygen"[All Fields] AND "therapy"[All Fields]) OR "hyperbaric oxygen therapy"[All Fields]) AND ("cognition"[MeSH Terms] OR "cognition"[All Fields] OR "cognitions"[All Fields] OR "cognitive"[All Fields] OR "cognitively"[All Fields] OR "cognitives"[All Fields]) AND ("ischaemia"[All Fields] OR "ischemia"[MeSH Terms] OR "ischemia"[All Fields] OR "ischaemias"[All Fields] OR "ischemias"[All Fields])

d) Ageing and neurodegenerative disorders Search: ((hyperbaric oxygen therapy) AND (neurodegenerative disease)) AND (cognition) ("hyperbaric oxygenation"[MeSH Terms] OR ("hyperbaric"[All Fields] AND "oxygenation"[All Fields]) OR "hyperbaric oxygenation"[All Fields] OR ("hyperbaric"[All Fields] AND "oxygen"[All Fields] AND "therapy"[All Fields]) OR "hyperbaric oxygen therapy"[All Fields]) AND ("neurodegenerative diseases"[MeSH Terms] OR ("neurodegenerative"[All Fields] AND "diseases"[All Fields]) OR "neurodegenerative diseases"[All Fields] OR ("neurodegenerative"[All Fields] AND "disease"[All Fields]) OR "neurodegenerative disease"[All Fields]) AND ("cognition"[MeSH Terms] OR "cognition"[All Fields] OR "cognitions"[All Fields] OR "cognitive"[All Fields] OR "cognitively"[All Fields] OR "cogni tives"[All Fields]) and Search: (hyperbaric oxygen therapy) AND (alzheimer disease) ("hyperbaric oxygenation"[MeSH Terms] OR ("hyperbaric"[All Fields] AND "oxygenation"[All Fields]) OR "hyperbaric oxygenation"[All Fields] OR ("hyperbaric"[All Fields] AND "oxygen"[All Fields] AND "therapy"[All Fields]) OR "hyperbaric oxygen therapy"[All Fields]) AND ("alzheimer disease"[MeSH Terms] OR ("alzheimer"[All Fields] AND "disease"[All Fields]) OR "alzheimer disease"[All Fields]) and Search: ((hyperbaric oxygen therapy) AND (neurodegeneration)) AND (cognition) Sort by: Most Recent ("hyperbaric oxygenation"[MeSH Terms] OR ("hyperbaric"[All Fields] AND "oxygenation"[All Fields]) OR "hyperbaric oxygenation"[All Fields] OR ("hyperbaric"[All Fields] AND "oxygen"[All Fields] AND "therapy"[All Fields]) OR "hyperbaric oxygen therapy"[All Fields]) AND ("nerve degeneration"[MeSH Terms] OR ("nerve"[All
Fields] AND "degeneration"[All Fields]) OR "nerve degeneration"[All Fields] OR "neurodegeneration"[All Fields] OR "neurodegenerating"[All Fields] OR "neurodegenerations"[All Fields]) AND ("cognition"[MeSH Terms] OR "cognition"[All Fields] OR "cognitions"[All Fields] OR "cognitive"[All Fields] OR "cognitively"[All Fields] OR "cognitives"[All Fields]) and Search: ((hyperbaric oxygen therapy) AND (ageing)) AND (cognition) Sort by: Most Recent ("hyperbaric oxygenation"[MeSH Terms] OR ("hyperbaric"[All Fields] AND "oxygenation"[All Fields]) OR "hyperbaric oxygenation"[All Fields] OR ("hyperbaric"[All Fields] AND "oxygen"[All Fields] AND "therapy"[All Fields]) OR "hyperbaric oxygen therapy"[All Fields]) AND ("aging"[MeSH Terms] OR "aging"[All Fields] OR "ageing"[All Fields]) AND ("cognition"[MeSH Terms] OR "cognition"[All Fields] OR "cognitions"[All Fields] OR "cognitive"[All Fields] OR "cognitively"[All Fields] OR "cognitives"[All Fields])

\section{Study Selection and Eligibility Criteria}

A two-step approach was used to select articles. Firstly, titles and abstracts of all search results were screened for the following characteristics (1) original article published in English, (2) case studies were excluded. Secondly, full-text articles were obtained from the selected studies and were reviewed on the following inclusion criteria (1) performed cognitive processes assessment (2) performed HBO therapy with described HBOT protocol. Two neuropsychologists independently reviewed titles, abstracts, full texts and extracted data. The review process is summarized in Figs. 1, 2, 3 and 4. Results from the systematic literature search are provided separately in each indication section of the manuscript.

\section{Results and Discussion}

\section{HBOT and Cognitive Dysfunctions After CO Intoxication}

Exposure to $\mathrm{CO}$, which excludes oxygen from tissues through the formation of a stable complex carboxyhaemoglobin $(\mathrm{COHb})$, can damage various body systems - including the CNS. The acute symptoms reported by patients are: headache, weakness or lethargy, dizziness, nausea, shortness of breath, chest pain, visual changes and muscle cramping. Moreover, up to $45 \%$ have cognitive problems with memory, attention and concentration (Weaver et al., 2002). Patients in more severe states (with a loss of consciousness or with $\mathrm{COHb}$ levels greater than 


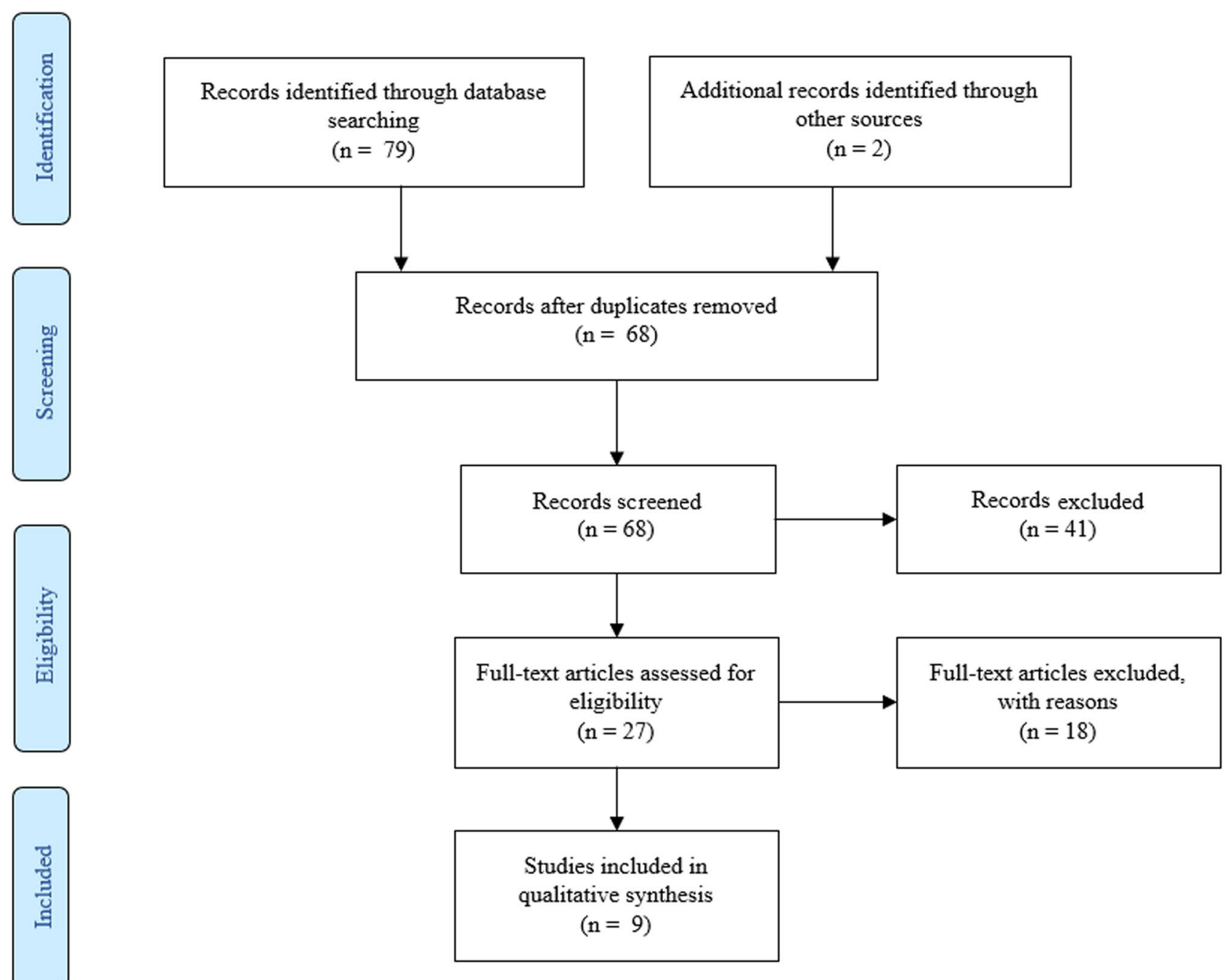

Fig. 1 PRISMA Flow Diagram for Included Studies for HBOT usage in cognitive dysfunction after CO intoxication

$25 \%$ ) frequently show cognitive dysfunction lasting 1 month or longer (Goldstein, 2008; Weaver et al., 2002). Multiple mechanisms are involved in both acute and delayed $\mathrm{CO}$ toxicity, including neuroinflammation, apoptosis and brain lipid peroxidation. All of them are ameliorated by HBOT (reviewed by Mannaioni et al., 2006).

Most patients with $\mathrm{CO}$ poisoning can recover from the acute phase. However, around $1-30 \%$ of patients develop delayed neuropsychological sequelae 2-6 weeks after recovery from the acute phase (Choi, 1983; Weaver, 2009; Weaver et al., 2007). Therefore, treatment for $\mathrm{CO}$ poisoning during the acute phase aims to address immediate threats to life as well as prevent delayed and sometimes permanent neuropsychological morbidity (Liao et al., 2019; Mathieu et al., 1985).

Gale and Hopkins (2004) recruited 20 patients with CO poisoning and assessed neuropsychological functioning.
They showed a correlation between impairment of verbal memory and visual memory and reduced hippocampal volume in patients. Cognitive disturbances were present even 6 months or longer after $\mathrm{CO}$ poisoning. Chang et al. (2010) described similar findings about persistent neuropsychological deficits (verbal episodic memory, visual memory and visual-spatial ability) in 9 patients with CO exposure: these issues were still present after 3 and 10 months. Moreover T2-weighted brain magnetic resonance imaging of patients with delayed neuropsychiatric sequelae after $\mathrm{CO}$ poisoning shows damage to the hippocampus (Bruno et al., 1993; Henke et al., 1999) and white-matter lesion in the frontal lobe and periventricular area (Mundy et al., 2013; Park \& Kim, 2014). Those regions are involved in reference memory and working memory, respectively (Burges et al., 2002; Prior et al., 1997). 
Fig. 2 PRISMA Flow Diagram for Included Studies for usage of HBOT in TBI-related cognition disorders
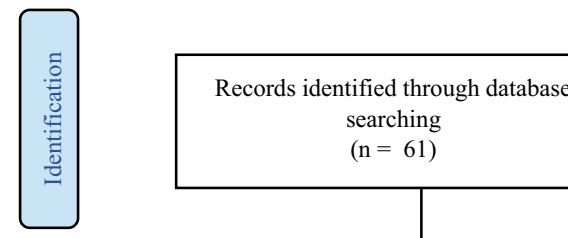
$(\mathrm{n}=61)$

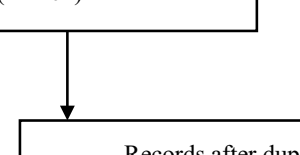

Records after duplicates removed $(\mathrm{n}=54)$

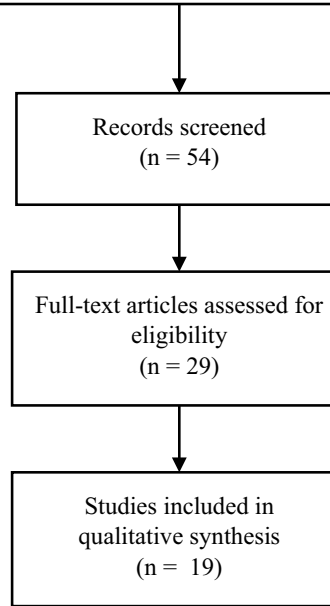

dissolved oxygen content, hastens the elimination of $\mathrm{COHb}$ in the blood and decreases cerebral oedema (Goldstein, 2008). HBOT is often recommended for patients with acute
Full-text articles excluded, with reasons $(\mathrm{n}=10)$
Additional records identified through other sources $(\mathrm{n}=13)$

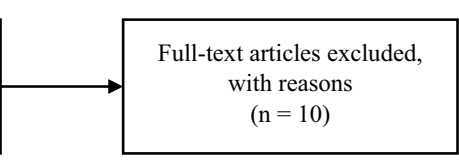

Fig. 3 PRISMA Flow Diagram for Included Studies for HBOT usage in postroke cognitive disturbances administration of $100 \%$ oxygen and general supportive care. Administration of supplemental oxygen inpeses the

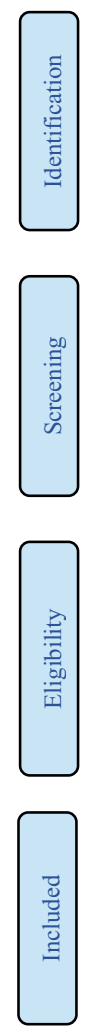

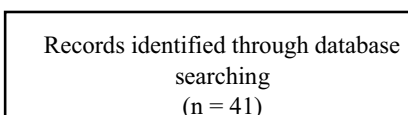

$(\mathrm{n}=41)$

$$
\text { tative synthes }
$$
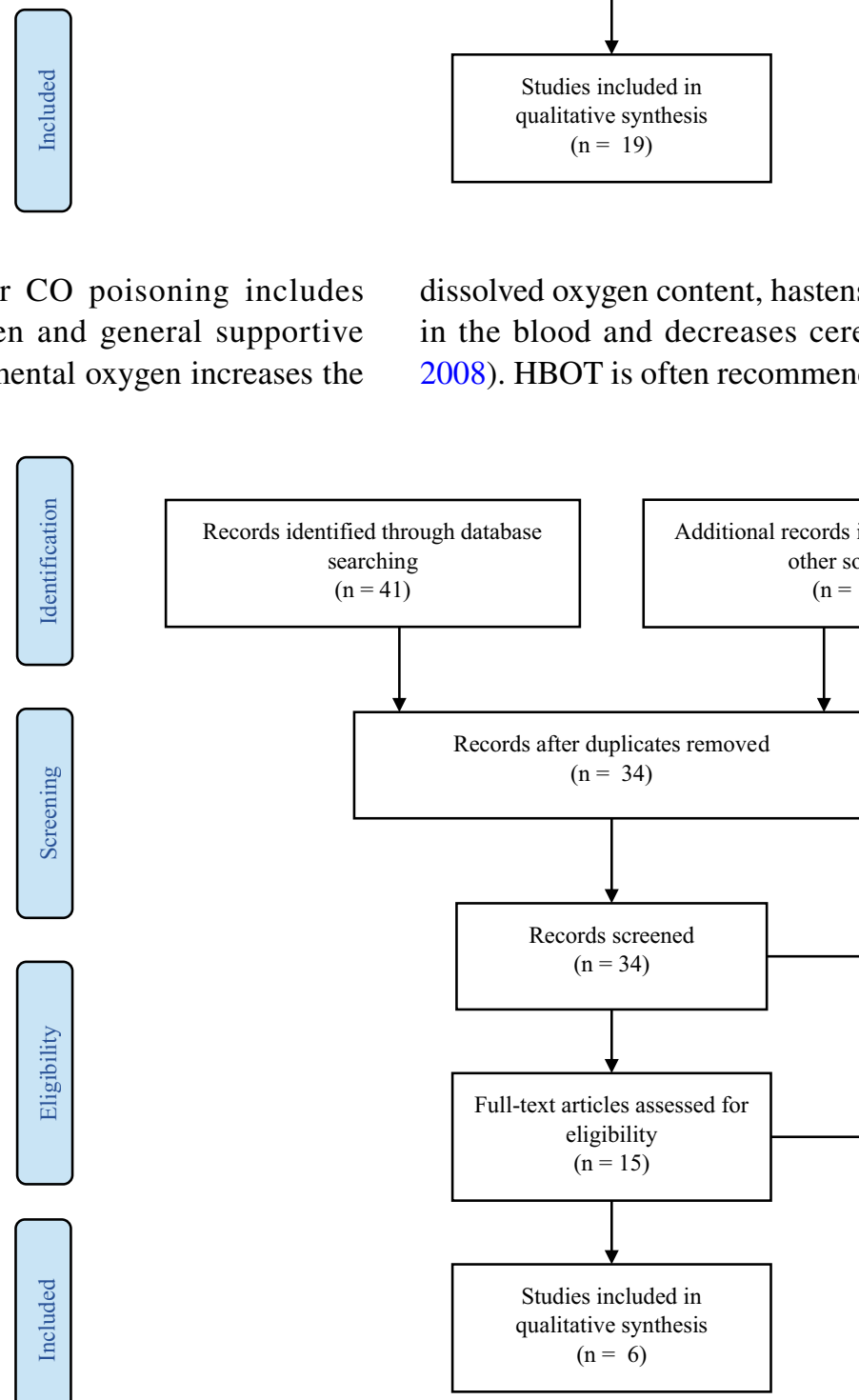

Additional records identified through other sources $(\mathrm{n}=13)$ 
Fig. 4 PRISMA Flow Diagram for Included Studies for HBOT in cognitive ageing and neurodegenerative disorders

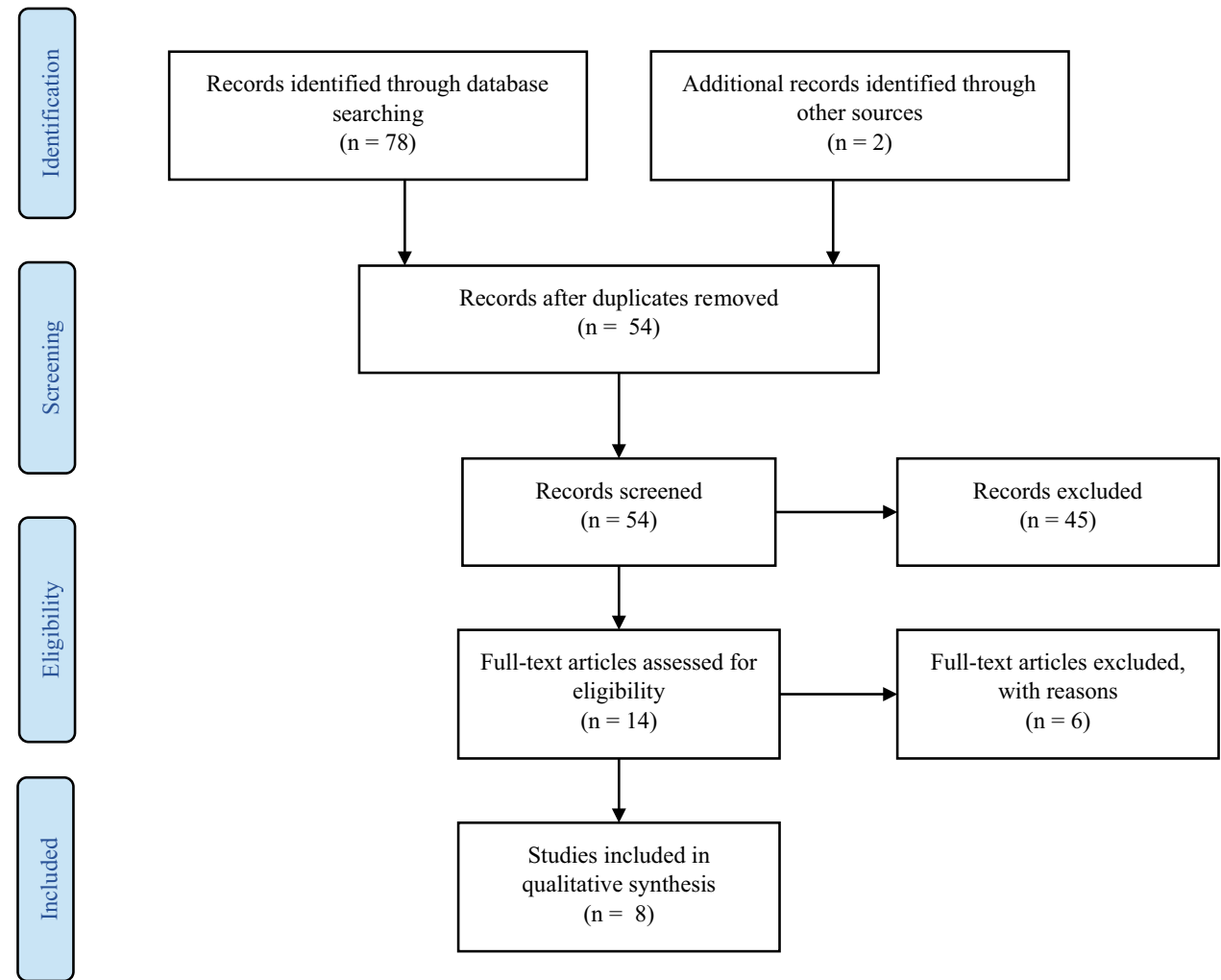

CO poisoning, especially if they have lost consciousness or have severe poisoning symptoms (Mathieu et al., 2017; Stoller, 2007).

There are also few animal studies in this field. Liu et al. (2016) sought whether neurogenesis is the target for HBOT to abrogate the delayed neuropsychological sequelae after CO poisoning. HBOT (2.5 ATA with $100 \%$ oxygen for $60 \mathrm{~min}$ ) was conducted on rats during the first 7 days after $\mathrm{CO}$ poisoning. Their animal research suggests that early HBOT may ameliorate delayed neuropsychological disturbances after acute $\mathrm{CO}$ poisoning by promoting neurogenesis through upregulating Brain-Derived Neurotrophic Factor (BDNF) in the hippocampus. Furthermore, the same group of authors conducted study with HBO therapy combined with N-butylphthalide on 80 rats. HBOT was conducted on the same conditions as in previous study. Rats performed Morris Water Maze Task. Last part of the study included hippocampus microstructure assessment. Authors concluded that combined therapy can improve cognitive functioning through maintaining ultrastructural integrity of hippocampus, and thus may play a neuroprotective role in brain tissue in rats with $\mathrm{CO}$ poisoning. Animal studies examining effect of HBOT on cognition after carbon monoxide intoxication are presented in Table 1.

Several studies have investigated the effect of HBOT with multiple neuropsychological methods. Scheinkestel et al. (1999) conducted a double-blind, randomised clinical trial to investigate the effects of HBOT by measuring cognitive functioning immediately after the therapy (daily treatments with $100 \%$ oxygen in a hyperbaric chamber $-60 \mathrm{~min}$ at 2.8 ATA for the HBOT group and at 1.0 ATA for the normobaric oxygen group - for three days) and 1 month later. They found no improvement in the patients' states, and they reported a worse outcome in learning tests.

Table 1 Effect of HBOT in carbon monoxide poisoning on cognition in animals

\begin{tabular}{lllll}
\hline Authors & Studied group & HBOT protocol & Cognitive measures & Results \\
\hline (Liu et al., 2016) & $\begin{array}{l}\text { 10 rats after acute carbon monoxide } \\
\text { poisoning }\end{array}$ & 7 sessions for 60 min at 2.5 ATA & Eight arm maze test & Improvement in memory \\
(Bi et al., 2017) & $\begin{array}{l}\text { 80 rats after CO intoxication; } \\
\text { divided in two groups HBOT and }\end{array}$ & 7 sessions for 60 min at 2.5 ATA & Morris Water Maze Task & Improvement in memory \\
& HBOT + N-butylphthalide & & & \\
\hline
\end{tabular}

$A T A$ absolute atmosphere, $C O$ carbon monoxide, HBOT hyperbaric oxygen therapy 
A similar methodology was used by Weaver et al. (2002); they examined 152 patients with CO poisoning with various neuropsychological tests after the first and third session and at 2 and 6 weeks, 6 months and 12 months after HBOT. All subjects underwent three protocol-directed sessions in monoplace hyperbaric chambers at intervals of 6 to $12 \mathrm{~h}$. The first oxygen treatment session was initiated within $24 \mathrm{~h}$ after the end of the exposure to $\mathrm{CO}$. During both hyperbaric-oxygen and normobaric-oxygen sessions, all intubated patients were mechanically ventilated with $100 \%$ oxygen. The patients in the HBOT group were exposed to $100 \%$ oxygen at 3 ATA and then 2 ATA during the first chamber session and then to $100 \%$ oxygen at 2 ATA during sessions 2 and 3 . Subjects in the normobaric-oxygen group were exposed to air at 1 ATA for all three chamber sessions. After three sessions, there were improvement in a few neuropsychological tests (Digit Span, Trail Making, Digit-Symbol, Block Design and Story Recall), demonstrating increased attention, visuo-spatial and memory processes as well as processing speed. This avoided two of the limitations of the study by Scheinkestel et al. (1999), i.e. loss to follow up and cluster rather than patient level randomisation for group exposures. Thus, it was the first study that was not plagued with or accused of having methodological flaws. From this study, 44 individuals were subsequently enrolled to ancillary prospective $A P O E$ genotyping study. The authors found that HBOT reduced cognitive deficits after $\mathrm{CO}$ poisoning in the absence of the e4 allele and concluded that HBOT is a useful treatment for preventing cognitive sequalae after $\mathrm{CO}$ poisoning (Hopkins et al., 2007).

Delayed neuropsychiatric sequel is estimated to occur in $10-30 \%$ of victims of carbon monoxide poisoning, but the reported incidence varies widely (Sönmez et al., 2018). Its various symptoms mainly comprise cognitive impairment, parkinsonism, urinary and faecal incontinence, dementia and psychosis (Choi, 1983; Tapeantong \& Poungvarin, 2009). Emergent HBOT within $24 \mathrm{~h}$ reduces the risk of cognitive sequelae after acute CO poisoning (Weaver et al., 2002).

Yeh et al. (2014) indicated that patients with delayed neuropsychiatric sequel after $\mathrm{CO}$ intoxication had poorer performance on general cognitive functioning, language skills, psychomotor speed, visual-spatial processes, logical and working memory and executive function compared to those with acute $\mathrm{CO}$ poisoning at 1 month. Compared with the acute neuropsychological sequel group, the group with delayed symptoms had more significant progress at 6-month follow-up after HBOT with regard to general cognitive function, psychomotor speed and visuo-spatial skills. However, the follow-up progress on language processes, logical memory and executive function tasks did not differ between the groups. These results support previous brain-image findings that patients with delayed neuropsychiatric sequel had abnormal deep white matter and frontal lobe regions (Ernst
\& Zibrak, 1998; Zagami et al., 1993). The authors suggested that future research should employ standardised and comprehensive neuropsychological tasks, as well as larger samples and matching brain-imaging investigations.

Lo et al. (2007) performed magnetic resonance, diffusion tensor imaging and Mini-Mental State Examination (MMSE) examination in 6 patients with delayed neuropsychiatric sequel immediately before and 3 months after HBOT (subjects underwent from 8 to 40 consecutive sessions, depending on the clinical response, with a pressure of 2.5 ATA for $120 \mathrm{~min}$ in each session) to obtain fractional anisotropy values and assess neuropsychological functioning. There was a significantly higher mean fractional anisotropy value in the control group compared with the patients both before and 3 months after HBOT. Notably, in the individuals with delayed symptoms of $\mathrm{CO}$ poisoning, the mean fractional anisotropy value 3 months after HBOT was also significantly higher than before HBOT. All of the patients regained full scores in the MMSE 3 months after the hyperbaric oxygen therapy.

Chang et al. (2010) examined 9 patients - with symptoms of delayed neuropsychiatric sequel - who received HBOT (at 2.5 ATA for 120 min five days per week during hospitalisation; 8-40 sessions). For all patients, cognitive symptoms significantly improved after the therapy, with significantly higher MMSE scores. However, white matter changes remained evident in the brain magnetic resonance scans.

Although HBOT has been applied clinically for the treatment of both acute and delayed phase of $\mathrm{CO}$ poisoning and HBOT reduces the neurological sequelae caused by the poison, the clinical benefits and the underlying mechanisms are still controversial (Annane et al., 2011; Birmingham \& Hoffman, 2011; Camporesi, 2014; Hampson et al., 2013; Weaver et al., 2007). Nevertheless, growing evidence has indicated that HBOT can promote proliferation of endogenous neural stem cells and stimulate neurogenesis in the injured brain (Lee et al., 2013; Mu et al., 2011; Zádori et al., 2011).

Delayed encephalopathy after acute $\mathrm{CO}$ poisoning (DEACMP) is a very serious complications (Goldstein, 2008). DEACMP is a common cause of clinical neurological complications and may result in memory impairment, unresponsiveness, visceral autonomic nervous system dysfunction, Parkinson's disease, cognitive dysfunction and behavioural disorders in patients. Currently, the pathogenesis of DEACMP remains elusive. Given that the CNS is the tissue that is most sensitive to oxygen, some researchers have sought primary factors that induce DEACMP in hypoxia caused by CO poisoning (Lee et al., 1988; Prockop \& Chichkova, 2007). This theory does not explain all clinical manifestations and pathological changes present in DEACMP, especially the variety of symptoms that occur after the recovery of the carbonyl haemoglobin level. Other researchers have reported that delayed $\mathrm{CO}$ poisoning neuropathology is associated with 
an adaptive immunological response to chemically modified myelin basic protein (Thom et al., 2004). Xiang et al. (2017a, b), in their double-blind, randomised study involving 215 DEACMP patients (MMSE score $\leq 24$, indicative of cognitive delay), found that both HBOT and the combined application of N-butylphthalide and HBOT (with 2.5 ATA for 80 min per day) significantly increased cognitive functioning (measured with MMSE) after short-term treatment. The efficacy of the combined treatment was greater than HBOT alone. After 8 weeks of treatment, almost half of the experimental group achieved significantly higher results compared to the control group. The researchers concluded that HBOT may increase the blood oxygen level to alleviate the hypoxic state in the brain of CO-poisoned patients. This phenomenon is beneficial to the functional recovery of damaged brain cells.

Moreover, the combined application of N-butylphthalide and HBOT may be a potential effective therapy in treating cognitive dysfunction for patients with DEACMP. Furthermore, the same authors conducted study on 120 DEACMP patients divided in two groups: HBOT alone and HBOT plus dexamethasone. Each patients received 20 hyperbaric oxygen therapy sessions at 2.5 ATA for 80 min per day. MMSE performed before and after 4 weeks of treatment showed cognitive improvement in both groups. Although authors concluded that the combined application of dexamethasone and HBO therapy should be considered as it yields better efficacy for patients with DEACMP (Xiang et al., 2017a, b). Efficacy of N-butylphthalide and dexamethasone combined with HBOT in patients with DEACMP was also studied in recent study by Zhang et al. (2020). Authors examined 171 DEACMP patients and divided in two groups: combined therapy and HBO alone. Cognitive state was assessed with MMSE and Montreal Cognitive Assessment (MoCA) scale before and 1 and 3 months and 1 year after the treatment. Their results indicate that combined therapy can significantly improve cognitive and motor functions of patients with DEACMP. Human studies examining effect of HBOT on cognition after carbon monoxide intoxication are presented in Table 2.

Current studies examining $\mathrm{CO}$ intoxication effects mostly agree that HBOT is promising therapy for improving patients cognition. Although research paradigm varies (e.g. therapy range from 3 to 40 HBOT sessions), thus comparing results should be made with caution. Furthermore, detailed neuropsychological assessment is rarely done. Cognitive screening methods (i.e. MMSE, MoCA) are not reliable and sensitive enough to detect subtle changes in cognitive functioning. Future studies should be designed to determine optimal combinations of the dose and timing of HBOT, and planned subgroup analyses should attempt to define which patients could benefit most from hospital transfer. These studies will hopefully provide the evidence needed to eliminate the remaining doubt about the effectiveness of HBOT. More aggressive and appropriate treatment options than are used today are needed. The correct diagnosis of the cognitive consequences of $\mathrm{CO}$ poisoning often occur quite a bit after the exposure. Thus, appropriate and timely treatment is even more problematic.

\section{HBOT and TBI-Related Cognition Disorders}

TBI can be caused by several factors. An external physical force, rapid acceleration or deceleration of the head, bleeding within or around the brain, lack of sufficient oxygen to the brain or toxic substances passing through the blood-brain barrier. The damage caused by TBI can be focal (confined to one area of the brain) or diffuse (involving more than one area of the brain: Zhang et al., 2014). Symptoms of a TBI vary from mild and moderate to severe, depending on the extent of the damage to the brain. TBI can result in temporary or permanent impairment of cognitive, emotional or physical functioning state. Thus, it has become a significant concern in civilian and military populations (Chiu \& LaPorte, 1993). TBI is linked with several pathological mechanisms, that is, diffuse shearing of axonal pathways and small blood vessels - which is also known as diffuse axonal injury (Medana \& Esiri, 2003) - ischaemia, mild oedema and other biochemical and inflammatory processes, that culminate in impaired regenerative or healing processes resulting from increasing tissue hypoxia (Zasler et al., 2007). Furthermore, TBI can lead to PCS, a complex of symptoms such as headaches, dizziness, imbalance, vertigo, fatigue, changes in sleep pattern, neuropsychiatric symptoms (e.g., behavioural and mood changes, confusion) and cognitive impairments (in memory, attention, concentration and executive functions) (McCauley et al., 2000). Due to multiple pathological mechanisms, cognitive impairments are usually the predominant symptoms localised in multiple brain areas (Kushner, 1998; Levin, 1990; Sohlberg \& Mateer, 2001a, b). The use of HBOT for brain injury is based on the hypothesis that injured or inactive neurons would benefit from increased blood flow and oxygen delivery, which would act to metabolically or electrically reactivate the cells (Deng, 2018; Francis \& Baynosa, 2017; Neubauer \& James, 1998).

In recent years, animal models (Chen et al., 2010; Efrati \& Ben-Jacob, 2014a, b; Lin et al., 2012; Neubauer \& James, 1998) and human studies (Barrett et al., 2004; Boussi-Gross et al., 2013; Golden et al., 2006; Harch et al., 2012; Shi et al., 2003; Tal et al., 2015; Wright et al., 2009) have shown that HBOT can improve PCS by targeting basic pathological processes (Hadanny \& Efrati, 2016). The evidence about the effectiveness of HBOT for TBI is conflicting, and the case series and time series studies of HBOT for TBI patients had serious flaws. One of the most concerning issues is the 


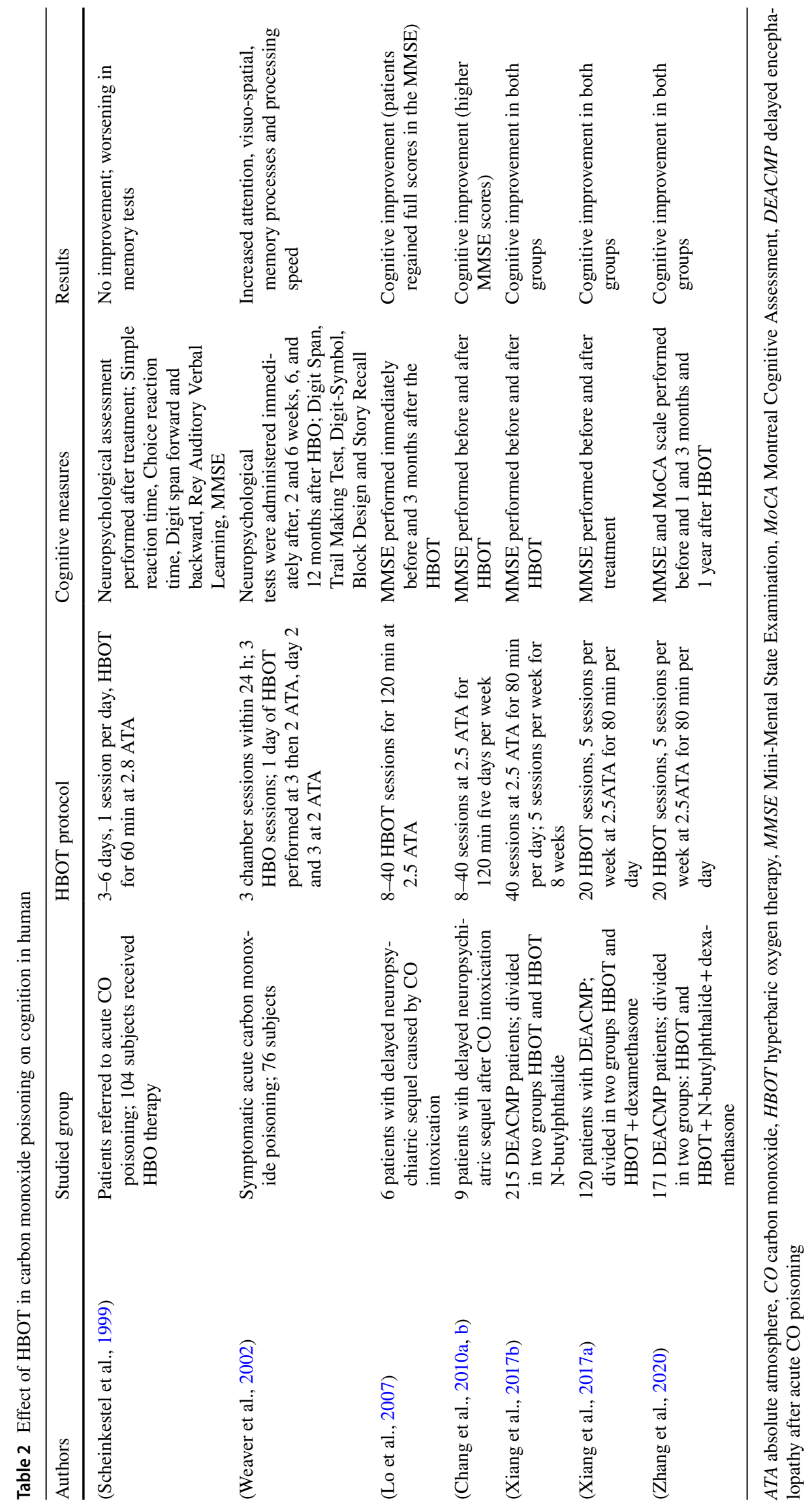


optimal time window for HBOT, a factor that can determine its efficacy in TBI.

Animal model studies have demonstrated that HBOT has a positive effect on cognitive outcome (Kraitsy et al., 2014; Lin et al., 2012). Zhou et al. (2007) found that rats with brain injury treated with HBO (1 h at 1.5 ATA + 3hour normobaric oxygen) had significant improvement in cognitive recovery HBO research using sham, NBO and HBO was used by Harch et al. (2007). Authors proved that 80 HBOT sessions performed twice a day for $90 \mathrm{~min}$ at 1.5 ATA can significantly improve spatial learning in TBI rats. They confirmed their results with showing increase in hippocampus vascular density after hyperbaric oxygen therapy. Wang et al. investigated the prolonged therapeutic time window of HBOT in animal models. Their study involved HBOT administration within $6 \mathrm{~h}$ after TBI. Their results showed decreased neuronal apoptosis and improved cognitive ability. Furthermore, they described that multiple HBOT sessions (3 ATA hourly for 3 or 5 days) could extend the therapeutic time window up to $48 \mathrm{~h}$ post-TBI (Wang et al., 2010). The authors also reported that a treatment initiated within $12 \mathrm{~h}$ after injury improved neurologic outcomes, compared with a longer window of $24 \mathrm{~h}$. They concluded that $72 \mathrm{~h}$ after injury there is no significant improvement after single a HBOT session. However, if the first HBOT starts at $24 \mathrm{~h}$ after concussion and continues for 3 or 5 consecutive days, there are significant improvements in cognitive deficits. Their results suggest that the optimal HBOT paradigm for human studies may be a single treatment initiated within $24 \mathrm{~h}$ after the injury, followed by treatments for 5 consecutive days. Liu et al. (2013) investigated the effect and mechanism of HBOT on cognitive functioning in rats. Authors suggest that hyperbaric oxygen therapy significantly improves spatial learning and memory skills at rats with traumatic brain injury, and the potential mechanism behind those improvements is mediated by metabolic changes and nerve cell restoration in the hippocampus. Another research proving neuroprotective effect of HBOT after TBI was conducted by Baratz-Goldstein et al. (2017). They investigated impact of 4 consecutive days hyperbaric oxygen treatment on mice with traumatic brain injury (2 different time lines: $3 \mathrm{~h}$ after injury and 7 days post injury). They found that mice treated with hyperbaric oxygen showed significant improvement in learning abilities. Their results suggest neuroprotective effect of HBOT in TBI with short and long therapeutic window. Similar results (memory improvement) were described by Chen et al. (2014). Moreover they described therapeutic effect of hyperbaric oxygen on neuroinflammation, apoptosis and oedema after TBI.

The data from these pre-clinic and clinic studies indicate that HBOT is beneficial when it is applied early after an insult or injury. Application of HBOT within a therapeutic time window established in preclinical study is an important requirement to ensure treatment efficiency. Studies examining effect of HBOT on cognition in animal models of TBI are presented in Table 3.

Cifu et al. (2014) conducted a randomised, blinded clinical study on subjects with persistent post-concussion symptoms. In their study - a randomised, controlled trial including 50 military service members suffering mild TBI between 3 and 71 months before HBOT. HBOT was assessed at 2.0 ATA. All

Table 3 Effect of HBOT in traumatic brain injury on cognition in animals

\begin{tabular}{|c|c|c|c|c|}
\hline Authors & Studied group & HBOT protocol & Cognitive measures & Results \\
\hline (Zhou et al., 2007) & 23 TBI rats & $\mathrm{HBO}$ for $1 \mathrm{~h}$ at $1.5 \mathrm{ATA}$ & $\begin{array}{l}\text { Morris water maze post } \\
\text { injury days } 11 \text { to } 15\end{array}$ & Improvements in cognition \\
\hline (Harch et al., 2007) & 19 TBI rats & $\begin{array}{l}80 \text { HBOT, twice a day, } \\
7 \text { days/week } 1.5 \text { ATA for } \\
90 \text { min }\end{array}$ & Morris water maze & $\begin{array}{l}\text { Improvement in spatial } \\
\text { learning }\end{array}$ \\
\hline (Wang et al., 2010) & 6 rats with TBI & $\begin{array}{l}3 \text { ATA hourly for } 3 \text { or } \\
5 \text { days }\end{array}$ & $\begin{array}{l}\text { Beam-balancing test; Pre- } \\
\text { hensile traction test }\end{array}$ & Improved cognitive ability \\
\hline (Liu et al., 2013) & 20 rats with TBI & $\begin{array}{l}\text { HBOT for } 60 \text { min daily at } 2 \\
\text { ATA for } 1 \text { and } 2 \text { weeks }\end{array}$ & $\begin{array}{l}\text { Morris water maze test } \\
\text { immediately after TBI, } 1 \\
\text { and } 2 \text { weeks of HBOT }\end{array}$ & $\begin{array}{l}\text { Improvement in spatial } \\
\text { learning and memory }\end{array}$ \\
\hline $\begin{array}{l}\text { (Baratz-Goldstein et al., } \\
\text { 2017) }\end{array}$ & 15 mice with TBI & $\begin{array}{l}\text { HBOT session for } 4 \text { days for } \\
60 \text { min at } 2 \text { ATA }\end{array}$ & $\begin{array}{l}7 \text { and } 30 \text { days after TBI; } \\
\text { Elevated plus maze, } \\
\text { Y-maze, Novel object } \\
\text { recognition test }\end{array}$ & $\begin{array}{l}\text { Improvement in learning } \\
\text { abilities }\end{array}$ \\
\hline (Chen et al., 2014) & $9 \mathrm{TBI}$ mice in each test & $\begin{array}{l}\text { HBOT performed } 3 \mathrm{~h} \text { after } \\
\text { injury for } 60 \mathrm{~min} \text { at } 2 \\
\text { ATA for } 5 \text { days }\end{array}$ & $\begin{array}{l}\text { Morris water maze (postin- } \\
\text { jury days } 14,15,16 \text { and } \\
17) \text {, beam walk task }(1,3,7 \\
\text { and } 14 \text { days post injury) }\end{array}$ & $\begin{array}{l}\text { Improved motor skills and } \\
\text { spatial learning }\end{array}$ \\
\hline (Zhou et al., 2007) & 23 TBI rats & $\mathrm{HBO}$ for $1 \mathrm{~h}$ at $1.5 \mathrm{ATA}$ & $\begin{array}{l}\text { Morris water maze post } \\
\text { injury days } 11 \text { to } 15\end{array}$ & Improvements in cognition \\
\hline
\end{tabular}


subjects were randomly assigned to one of three groups, breathing $10.5 \%, 75 \%$ or $100 \%$ oxygen to mimic normal air at 1.0 ATA, $100 \%$ oxygen at 1.5 ATA or $100 \%$ oxygen at 2.0 ATA, respectively. HBOT at either 1.5 or 2.0 ATA equivalent had no effect on post-concussion symptoms after mild TBI when compared with sham compression. However, the HBOT effect on cognitive functions was assessed with the self-administered Rivermead Post-Concussion Symptoms Questionnaire (RPQ), which is known to display several flaws in implementation and in its ability to accurately reflect test-taker experience. Moreover, interpretation and accuracy of the RPQ can vary widely due to self-administration and the various confounding variables involved. Indeed, it is sensitive to subjective patient memory, social desirability, stress and other covariates such as personality factors and willingness to reveal problems, as are the two other methods. Relying completely on the self-administration assessments is a weakness of this study (Potter et al., 2006).

Furthermore, Walker et al. (2014) conducted a randomised, double-blind and sham-control feasibility trial comparing pretreatment and posttreatment conducted in 60 male active-duty marines with combat-related mild TBI. Subjects with PCS that had persisted for 3 to 36 months were randomised to one of three groups receiving preassigned oxygen fractions $(10.5 \%, 75 \%$ or $100 \%)$ at 2.0 ATA. This design led to groups with an oxygen exposure equivalent to breathing surface air, 100\% oxygen at 1.5 ATA, and 100\% oxygen at 2.0 ATA, respectively. Each subject received 40 hyperbaric chamber sessions of 60 min each for 10 weeks. Multiple neuropsychological tests of cognitive performance were collected preintervention and 1-week postintervention. There were no significant changes in cognitive functioning between groups and pre and post HBOT. The authors concluded that HBOT is not useful to treat cognitive, balance or fine motor deficits associated with mild TBI and PCS.

Harch et al. (2012) performed an uncontrolled HBOT trial of 16 participants with PCS after blast exposure during military service. There was an improvement in both cognitive and psychomotor characteristics. The authors reported significant improvement one week after HBOT (40 at 1.5 ATA, 5 days per week for $60 \mathrm{~min}$ each session) in full-scale intelligence quotient (IQ), delayed and working memory, executive functions and attention. In a randomised trial, Wolf et al. (2012) studied participants with chronic PCS. Their results demonstrated no efficacy in cognitive impairment treatment with HBOT at an exposure pressure of 2.4 ATA for $90 \mathrm{~min}$ given once daily for 30 treatments compared to 1.3 ATA air exposure. However, both groups (the control group received treatment with room air at 1.3 ATA) improved beyond what would be expected more than 6 months after mild TBI. In subsequent study, they received similar results - no significant difference was observed between air (1.3 ATA) and HBO (2.4 ATA) although both groups shoved improvement in cognition (without statistical significance). The Wolf et al. (2012) studies were widely criticised for using 1.3 ATA in control group (Mychaskiw \& Stephens, 2013; Weaver et al., 2013). Actually, it should be recognized that 1.3 ATA air and 2.4 ATA oxygen may represent low and high edges of the oxygen dose-response curve. Hyperbaric physiology indicates that relatively subtle changes in tissue partial oxygen pressure may exert a significant therapeutic effect (Mychaskiw \& Stephens, 2013; Weaver et al., 2013).

Boussi-Gross et al. (2013) presented a prospective, randomised and controlled crossover study of the effect of HBOT with $100 \%$ oxygen at 1.5 ATA (5 days per week, 60 min each) on mild TBI patients at late chronic stage. The authors randomly divided the subjects into treated or crossover groups. The patients in the treated group were evaluated before and after 40 HBOT sessions. Subjects in the control group were evaluated at three times, baseline, and after 2 and 4 months. Neuropsychological examination included assessment of information processing, attention, memory and executive functions. All subjects underwent single photon emission computed tomography examination. HBOT induced neuroplasticity and significant brain function improvement in mild TBI patients with prolonged PCS (at a late chronic stage, years after brain injury). There was also improvement in all assessed cognitive functions. Moreover, changes in single photon emission computed tomography images after treatment indicate that HBOT reactivates neuronal activity in stunned areas that seem normal under computed tomography and magnetic resonance imaging.

Tal et al. (2015), in their study using perfusion magnetic resonance imaging, proved that HBOT can significantly increase cerebral blood flow and cerebral blood volume following 50-70 daily hyperbaric sessions, 5 days a week (each session consisted of $60 \mathrm{~min}$ of exposure to $100 \%$ oxygen at 1.5 ATA). There was also significant cognitive improvement in patients post TBI. The mean time from the acute injury was $10.3 \pm 3.2$ years. The most prominent improvements were seen in information processing speed, visual spatial processing and motor skills indices. The increased perfusion to the dysfunctional tissue, and the significant cognitive improvement, suggest that impaired tissue perfusion may serve as a rate limiting factor for regeneration and neuroplasticity even years after the acute injury. The authors concluded that that appropriate biological trigger can induce neuroplasticity months to years after the acute injury.

In a subsequent, study Tal et al. (2017) evaluated diffusion tensor imaging changes before and after HBOT of prolonged PCS. HBOT was initiated 6 months to 27 years $(10.3 \pm 3.2$ years $)$ from injury for 60 daily hyperbaric sessions, 5 days per week with sessions consisting of $90 \mathrm{~min}$ of exposure to $100 \%$ oxygen at 2 ATA. The authors found an increase in fractional anisotropy and a decrease in mean diffusivity after HBOT, together with cognitive function improvement of patients in the late chronic stage of TBI. 
Their results suggest that HBOT can induce brain microstructure recovery with significant improvement in memory, executive functions and information processing speed. They concluded that HBOT can improve the integrity of brain fibres, a phenomenon that correlates with improved cognitive functioning. It should be kept in mind, however, that quantitative validation of diffusion tensor imaging pathologic metrics remains very limited (Winklewski et al., 2018).

Churchill et al. (Churchill et al., 2016) examined effect of HBOT on information processing speed after mTBI. In their study, investigators measured speed of processing at baseline, 6 and 13 weeks in military personnel with mTBI. Subjects were randomized to 40 sessions of air (1.2 ATA) or HBOT (1.5 ATA). They found no significant changes in reaction time between $\mathrm{HBO}$ or air.

Furthermore Shandley and colleagues found that HBOT (90 min at 2.4 ATA) correlates with stem cell mobilization as well as increased neuropsychological performance comparing to $90 \mathrm{~min}$ air at 1.3 ATA. Authors suggest that stem cell mobilization may be required for cognitive improvement in TBI population.

Hadanny et al. (2018) demonstrated the neurotherapeutic effects of HBOT for chronic TBI. The study included 154 subjects $(42.7 \pm 14.6$ years $)$ with documented TBI 0.3-33 years (mean $4.6 \pm 5.8$, median 2.75 years) prior to HBOT. The HBOT protocol comprised 40-70 daily hyperbaric sessions, 5 days a week. Each session involved exposure to $100 \%$ oxygen at $1.5 / 2$ ATA. The authors confirmed that HBOT induced significant improvement in memory, executive functions, information processing speed and global cognitive scores.

Weaver et al. (2012) proposed the design of randomised study to evaluate the efficacy and utility of HBOT for American combatants with PCS. Based on this design authors conducted clinical trial. They examined executive functions, processing speed, memory, and learning of 71 military service members with mTBI (35 with PTSD) who were randomly administered to $\mathrm{HBO}$ or air (40 HBO sessions at 1.5 ATA vs air at 1.2 ATA for $60 \mathrm{~min}$ ). Authors found that after 13 weeks HBOT improved post-concussive and PTSD symptoms, cognitive processing speed, sleep quality, and balance function, especially in individuals with PTSD. Although changes did not persist beyond six months (Weaver et al., 2018). Research on effect of HBOT on cognition in traumatic brain disorders in humans are presented in Table 4.

Currently, the results of HBOT in clinical TBI trials are controversial, and the efficiency of HBOT in TBI has not been well established. First, the optimal time window for HBOT administration must be determined to ensure its efficacy in treating TBI. Second, objective and precise neuropsychological assessment methods are another challenge in the evaluation of the efficacy of HBOT in TBI patients. Third, heterogeneity in patients and HBOT paradigms (pressure, frequency, length of treatment course) partly affect or determine the outcome. There have been variations in patients' age and in the severity and nature of the injury in the studies. Future trials of HBOT for PCS should consider measuring outcomes with standardised neuropsychological methods and at longer intervals postintervention or in combination with rehabilitation therapy to determine potential delayed or priming effects.

\section{HBOT and Poststroke Cognitive Disturbances}

Stroke is a result of a blocked artery or a ruptured blood vessel. It leads to an interruption in cell homeostasis and symptoms such as loss of speech and loss of motor function. It is a major cause of disability and mortality among adults, with long-term impairments in the physical, emotional and cognitive state of survivors (Robinson, 2006). Neuropsychological disturbances after stroke are very common; they involve multiple cognitive deficits that lead to a decline in everyday functioning and in social functioning (Godefroy $\&$ Bogousslavsky, 2007). The main therapeutic targets are the regions surrounding the focal site of injury where the tissue is at high risk of disruption but not irreparably damaged; thus, there is still the potential to salvage these neurons (Baron, 2001; Lo et al., 2003; Singhal, 2007). Cell death and reduced neuronal activity caused by an ischaemic event can lead to excitotoxicity, oxidative stress, inflammation and apoptosis, all of which are pathways where hypoxia plays a key role (Lo et al., 2003). Therefore, increased oxygenation has been considered as a potential treatment for stroke; this treatment may lead to tissue repair and the generation of new synaptic connections (Golden et al., 2002; Neubauer \& James, 1998). Therapy and neuropsychological rehabilitation programmes are valuable for improving cognition at early stages, but they usually provide only partial recovery from symptoms. To date, there is no efficient neuropsychological rehabilitation programs available for late chronic stages (Rajeswaran, 2013; Ricker \& Callahan, 2000).

Current concepts of the pathophysiology of stroke provide a rationale for using HBOT in its management. Conventional methods of stroke treatment and their functional consequences are not satisfactory, and the outcomes remain controversial. There are only a few experimental animal studies and uncontrolled human trials that have shown the effectiveness and safety of HBOT after stroke. Clinical observations and basic research data suggest that HBOT may be a useful and effective treatment option in the management of acute stroke, but more studies are needed to clarify its clinical utility (Sánchez, 2013). The use of HBOT as a treatment following stroke was first raised 50-60 years ago (Hart \& Strauss, 2003; Hart \& Thompson, 1971). Despite decades of interest, studies that have investigated the effects of HBOT following a stroke have produced mixed results (Bennett et al., 2014; Freiberger et al., 2016; Helms et al., 


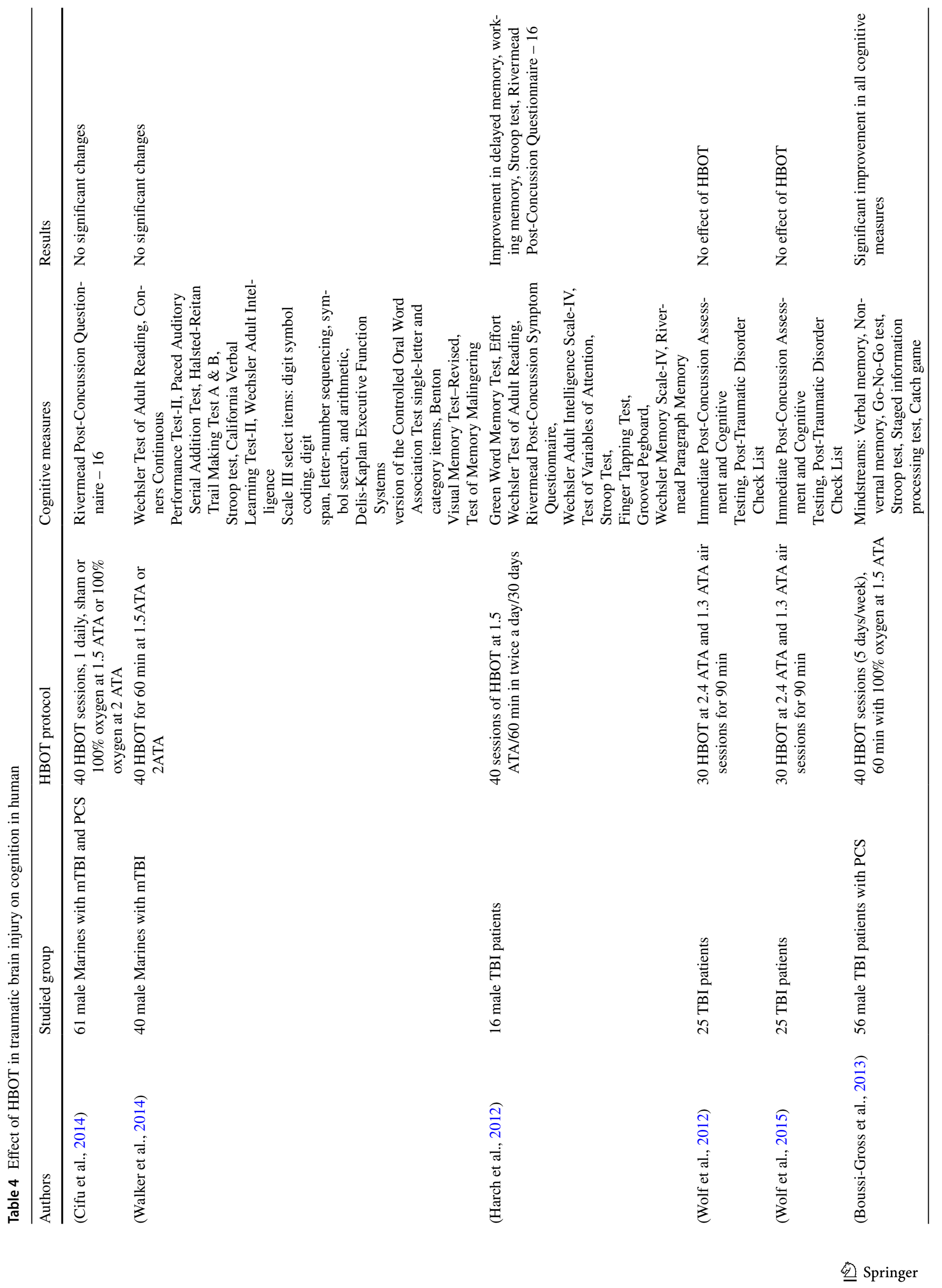




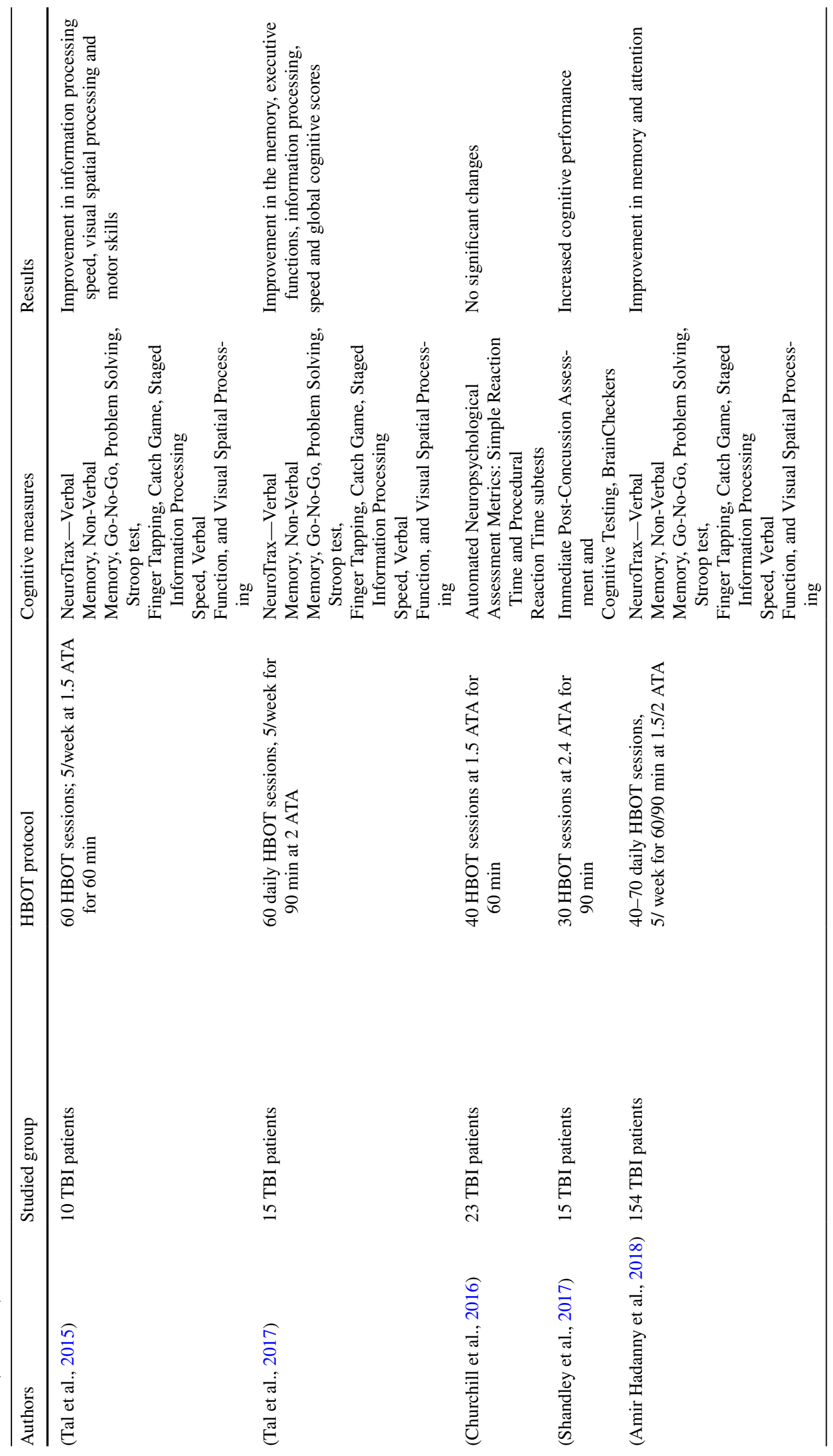


2005). Rehabilitation of stroke patients is one aspect that should be planned during the first few months following stroke. Thus, long-term follow-up studies are required to determine whether such measures would reduce the chronic disability attached to impaired cognition in stroke in stroke patients.

First clinical researches in this field were made by Sarno and Sarno (Sarno et al., 1972a, b). Authors investigated the effect of HBOT on language skills disrupted after stroke. First study included 16 left hemisphere stroke patients, second: 32 stroke patients ( 16 with left sided damage). Patients underwent neuropsychological examination, with detailed assessment of various verbal processes, before chamber treatment and after every session. Each patient participated in two double blinded conditions: HBOT (performed at 2 ATA of $100 \%$ oxygen) or sham (10.5\% oxygen both for $90 \mathrm{~min}$ ) with random order. Authors found no significant changes in neuropsychological tests results after exposure to oxygen.

Boussi-Gross et al. (2015) presented a retrospective analysis of the effects of HBOT on memory impairments in poststroke patients during the late chronic, unremitting stage. The HBOT protocol consisted of 40 to 60 daily sessions, 5 days per week, 90 min each, $100 \%$ oxygen at 2 ATA. Their data showed statistically significant improvements in memory functions in the majority of patients. These neuropsychological findings were in good agreement with metabolic brain changes assessed by single photon emission computed tomography brain imaging. Imaging analysis identified the brain regions associated with the memory impairments and improvements (perirhinal cortex and its activation correlated with clinical improvement in the delayed memory measures; improvement in verbal and nonverbal delayed memory abilities with the change in activation in the left and right perirhinal cortex, respectively).

These results are consistent with previous reports that HBOT induces neuroplasticity effects at late chronic stage, although these studies lacked a control group. Nevertheless, these and previous studies have provided convincing evidence that HBOT can induce neuroplasticity at chronic poststroke stages in areas with metabolic dysfunction, which if relevant to memory function in the brain can improve after HBOT (Boussi-Gross et al., 2013; Efrati et al., 2013).

Hadanny et al. (2015) firstly examined effect of HBOT on patients suffering from anoxic brain damage caused by cardiac arrest. Patients received 60 daily HBOT sessions of $100 \%$ oxygen at 1.5 ATA for 60 min. Neuropsychological measures were compared with single photon emission computed tomography results. Authors found significant improvement in memory, attention and executive functions. Those changes correlated with increased brain activity in relevant brain areas assessed by single photon emission computed tomography imaging. Their further retrospective research 
focus was put on 162 stroke patients (87 in left hemisphere, 121 ischemic). They found that HBOT (40-60 sessions, 90 min of $100 \%$ oxygen at 2 ATA) had significant effect on all cognitive domains. Authors concluded that hyperbaric oxygen therapy can be successful treatment even in late chronic stage of post-stroke patients (Hadanny et al., 2020).

In one of the most recent studies, Rosario et al. (2018) measured the impact of HBOT across a number of cognitive domains, including speech, language skills, general cognition, memory and emotional/behavioural impairments. The authors assessed functional abilities over a 3-month period for 6 subjects who underwent two 4-week periods of HBOT (commenced over 6 months after a stroke). HBOT comprised 20 total treatments of $100 \%$ at 2.0 ATA for $60 \mathrm{~min}$ for 4 weeks (on weekdays). There were significant improvements in memory and executive function after oxygen therapy. Despite a small sample size, the authors concluded that their findings support the idea of HBOT as a potential intervention following stroke. Researches on effect of HBOT on cognition after ischemia/stroke are presented in Table 5.

Clearly, additional larger prospective, randomised trials on the effect of HBOT on cognitive impairment during acute and delayed poststroke periods should be conducted. Moreover, future studies should widen the assessment of the HBOT effects on different cognitive functions because most of the existing studies focus on memory abilities. Additional study limitations relate to the HBOT protocol. Even though they have shown similar, significant beneficial effect, studies that have evaluated HBOT in stroke management have used different treatment protocols. The exact HBOT protocol that will induce maximal neuroplasticity with minimal side effects must be determined. Bennet et al. (2014) reported that when taken together, the existing literature does not indicate that HBOT is an effective intervention in the acute phase following an ischaemic stroke. Nevertheless, the failure of some clinical stroke trials that have utilised HBOT is probably linked to factors such as delayed time to therapy, inadequate sample size and the use of excessive chamber pressures (Singhal, 2007). Further research could focus on investigating HBOT effects on cognitive functions on stroke patients receiving thrombolysis or thrombectomy treatment before oxygen therapy.

\section{HBOT Usage for Cognitive Ageing and Neurodegenerative Disorders}

Dementia is a condition characterised by increasing several cognitive deficits such as loss of memory, problems with speech and understanding and visuo-spatial disruption. According to the 2003 World Health Organization (WHO) World Health Report, dementia causes $11.2 \%$ more years lived with disability than cardio- and cerebrovascular diseases and all forms of cancer in people aged 60 years and older. There are currently around 36 million dementia patients worldwide. It is anticipated that the number of dementia cases will increase in the subsequent years and will reach 81.1 million by the year 2040 (Ferri et al., 2005). Research on HBOT for dementia has mainly focussed on animal experiments (Table 6).

The first investigation of HBOT in the elderly population was performed by Jacobs et al. (1969). They reported improved cognitive functioning in 13 elderly patients with chronic organic brain syndrome after exposure to hyperbaric oxygen (30 sessions, twice a day with $100 \%$ oxygen at 2.5 ATA). Five control subjects exposed to a neutral air mixture failed to show improvement. Over the next six years, five other research reports were published. Three confirmed Jacobs original observation (Edwards \& Hart, 1974; Jacobs et al., 1972; Raskin et al., 1978), while two did not (Goldfarb et al., 1972; Thompson et al., 1976). The study by Goldfarb et al. (1972) was performed on 10 patients (mean age 74 years) with cognitive decline. All subjects underwent 40 to $58 \mathrm{~h}$ of exposure to $100 \%$ oxygen at 2.5 ATA in two sessions per day ( 90 min each). Compression and decompression time of each individual chamber session was about $110 \mathrm{~min}$. Neuropsychological examination was performed before and after 15 days of treatment. There were no significant improvements in cognitive outcome of subjects. Moreover Thompson et al. (1976) included 21 subjects with dementia (50-80 years old, 13 with diagnosis of cortical atrophy, 8 cerebrovascular disease) and 4 control subjects. HBOT consisted of the same procedure as Jacobs et al. (1969). There were no significant differences between the experimental and control subjects (Jacobs et al., 1972), although the severity of dementia in subjects in both studies was different. The patients in the Jacobs study had less cognitive deterioration. The interest in treating dementia with HBOT has grown in recent years.

Alzheimer disease is the most common form of dementia. It is characterised by progressive cognitive impairment and psychobehavioural disturbances. In a rodent model of Alzheimer disease, Shapira et al. (2018) found that HBOT can improve cognitive function by reducing neuroinflammation. The authors conducted their study with 6 mice (3 HBO treated, 3 nontreated). For animals in the treated group, HBOT was administered as $100 \%$ oxygen at a pressure of 2 ATA for 60 min daily for 14 consecutive days. The effects of HBOT on cognitive functions (memory and behaviour) in mice were evaluated using a series of behavioural tests. Cognitive tests were performed during the 7 days preceding sacrifice with a 24-h delay after the last HBOT or control treatment and a 48-h delay after the last task to reduce stress. The authors concluded that HBOT can ameliorate Alzheimer disease pathology and behavioural deficits in a transgenic mouse model of Alzheimer disease. Furthermore, in another rodent model of Alzheimer disease, Zhao et al. (2017) proved that HBOT can reduce hippocampal neuronal 


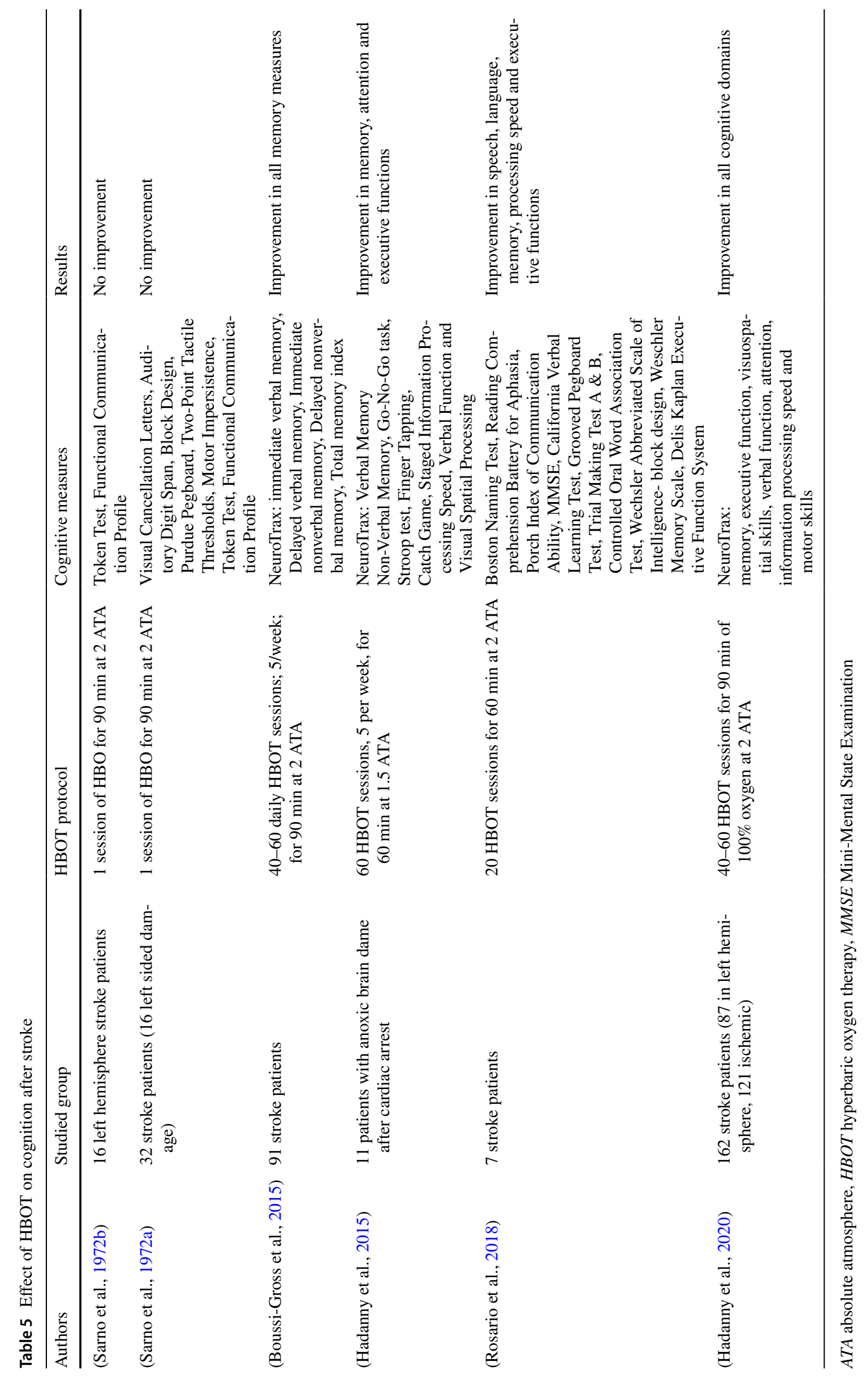


Table 6 Effect of HBOT on cognition in animal models of neurodegenerative disease

\begin{tabular}{|c|c|c|c|c|}
\hline Authors & Studied group & HBOT protocol & Cognitive measures & Results \\
\hline (Shapira et al., 2018) & $\begin{array}{l}14 \text { old triple-transgenic } \\
\text { mice and } 14 \text { non trans- } \\
\text { genic }\end{array}$ & $\begin{array}{l}\text { HBOT } 100 \% \text { oxygen at } 2 \\
\text { ATA for } 60 \text { min daily for } 14 \\
\text { consecutive days }\end{array}$ & $\begin{array}{l}\text { Y-maze, Open field test, } \\
\text { Novel object recognition } \\
\text { test }\end{array}$ & $\begin{array}{l}\text { Improvement in cognition and } \\
\text { behaviour measures }\end{array}$ \\
\hline (Zhao et al., 2017) & 8 rats with $\mathrm{AD}$ & $\begin{array}{l}5 \mathrm{HBO} \text { sessions at } 2 \text { ATA for } \\
60 \mathrm{~min}\end{array}$ & Morris water maze task & $\begin{array}{l}\text { Improvement in learning and } \\
\text { memory }\end{array}$ \\
\hline (Zhang et al., 2010) & $10 \mathrm{VaD}$ rats & $\begin{array}{l}10 \text { HBOT sessions for } 90 \mathrm{~min} \\
\text { at } 2 \text { ATA }\end{array}$ & The one-way avoidance test & $\begin{array}{l}\text { Improvement of learning and } \\
\text { memory }\end{array}$ \\
\hline
\end{tabular}

$A D$ Alzheimer Disease, $V a D$ Vascular Dementia, ATA absolute atmosphere, HBOT hyperbaric oxygen therapy

apoptosis and thus improve cognitive function. The authors examined 24 rats ( 3 groups: 8 normal, 8 Alzheimer disease, 8 Alzheimer disease $\mathrm{HBO}$ ) that underwent the experimental procedure. The Alzheimer disease HBOT group received six HBOT sessions (2 ATA) for $60 \mathrm{~min}$, while the normal and Alzheimer disease groups were placed in hyperbaric chambers without compression or decompression treatment. The authors concluded that hyperbaric treatment improves learning and memory skills by inhibiting dendritic spine loss and reducing neuronal apoptosis, astrocyte activation and tumour necrosis factor $\alpha$ (TNF- $\alpha)$ production in the hippocampus of rats with Alzheimer disease.

The second most common form of dementia is vascular dementia, accounting for approximately $30 \%$ of all cases of dementia (Kalaria et al., 2008). Vascular dementia is group of syndromes based on varying vascular mechanisms, such as multiple infarcts, small vessel ischaemic disease, strategically placed infarcts, hypoperfusion and haemorrhage, Alzheimer disease with cerebrovascular disease, hereditary vascular dementia or cerebral autosomal dominant arteriopathy with subcortical infarcts and leukoencephalopathy (Brien et al., 2003). To date, no effective treatments have been approved for established vascular dementia. Current treatment methods focus mainly on the reduction of risk factors and slowing the progression of the clinical outcome (Erkinjuntti et al., 2004; Moorhouse \& Rockwood, 2008; Sorrentino et al., 2008). A study on animal models of vascular dementia demonstrated that HBOT (2 ATA for $120 \mathrm{~min}$ [pressure boost for $15 \mathrm{~min}$, steady pressure for $90 \mathrm{~min}$, decompression for $15 \mathrm{~min}$ ] once a day for 10 continuous days) improves the blood supply and promotes neurogenesis in the piriform cortex (Zhang et al., 2010). These observations suggest possible benefits of HBOT for vascular dementia in humans.

Cerebrovascular disease includes a variety of conditions that affect the brain and the cerebral circulation which can lead to vascular dementia (Kuźma et al., 2018). Vila et al. (2005) examined possible reversible effect of HBOT on cognitive decline in cerebrovascular disease. Their study included 26 subjects with mild to moderate leukoaraiosis
(18 received 10 sessions of HBOT for $45 \mathrm{~min}$ at 2.5 ATA, 8 air sessions at 1.1 ATA). After treatment subjects receiving hyperbaric oxygen showed significant improvement in motor and cognitive scales compared to control group.

Wang and colleagues (Wang et al., 2009) examined 64 vascular dementia patients (32 HBOT, 32 control subjects) who underwent 12 weeks of HBOT sessions (97\% oxygen administered at 2.0 ATA for $60 \mathrm{~min}$ a day for 24 consecutive days a session; six days of rest in between sessions) as an adjuvant treatment to donepezil. Patients who received oxygen treatment showed better cognitive function compared to the control group treated with donepezil alone, measured either by the MMSE or by Hasegawa's Dementia Rating Scale.

Similar methodology was used by Xu et al. (2019). Authors prospectively analysed 158 vascular dementia patients who were randomized in two groups: HBO treatment (12 weeks of HBOT sessions administered at 2 ATA for $60 \mathrm{~min}$ ) and control group, both treated with donepezil hydrochloride. Authors concluded that HBO therapy can improve cognitive functions in patients with vascular dementia.

In one of the most recent reviews in this field, You et al. (2019) described HBOT as effective and safe complementary therapy for the treatment of vascular dementia. However, more research is needed because the exact mechanism of this treatment modality remains unclear. Given that HBOT has shown usefulness in treating a variety of conditions, the possible efficacy for treating Alzheimer disease and vascular dementia should be considered in experimental and preliminary clinical studies. Effect of hyperbaric oxygen therapy on dementia is presented in Table 7.

Future research should consider more detailed cognitive assessment. The current methodology is based mostly on brief neuropsychological batteries and tests, for example, the MMSE and MoCA. Furthermore, research must find the most effective HBOT protocol and include more subject and control groups. Moreover, multimodal assessment of positron emission tomography and magnetic resonance imaging networks could provide additional information on the impact of HBOT on neurodegenerative diseases. 


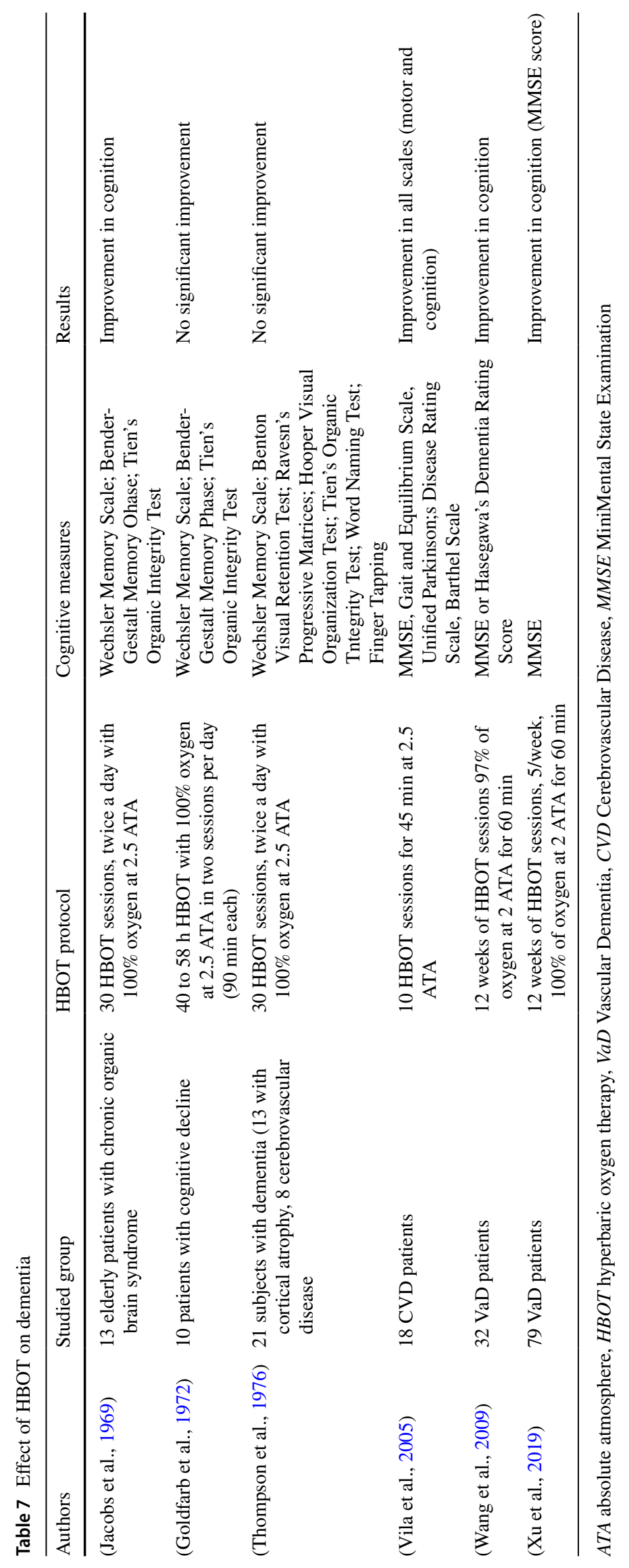




\section{Conclusions}

To the best of our knowledge this review provides the first state-of-the art, systematic summary of research focused on the use of HBOT in various neurological conditions.

Existing clinical data can be conflicting due to several inherent procedural issues, such as the use of non-objective endpoints, the lack of appropriate brain imaging as part of the inclusion criteria, inappropriate placebo of a hyperbaric environment as well as the inclusion of patients and lack of control groups. Clearly, larger randomised trials that evaluate the effect of HBOT on cognitive impairment should be conducted. Current HBOT studies have reported controversial results with regard to the efficiency of HBOT in various neurological conditions with cognitive disturbance outcome.

Some study limitations relate to HBOT itself. There is still no agreement about an HBOT protocol, specifically the appropriate air pressure and the time and repetition of treatment sessions. The exact HBOT protocol for each neurological state - which will induce maximal neuroplasticity and functional improvement with minimal side effects - must be determined.

Future studies should widen the assessment of HBOT effects on different cognitive functions because most of the existing investigations have focussed on single processes. Furthermore, more objective and precise neuropsychological assessment methods are needed to evaluate the efficacy of HBOT for neuropsychological deficits. To reach an agreement about the effectiveness of HBOT for neuropsychological disorders, research needs to focus more on homogeneity of the included subjects. The current studies were conducted using heterogeneous groups of patients with wide variations in age, severity and nature of the brain damage. Thus, there is a need for longitudinal studies to verify whether the administration of a more extensive series of HBOT sessions will lead to longer-lasting improvements in cognitive functioning.

Assuming that the methodological issues described in this review can be properly addressed and evaluated HBOT may have potential for the treatment of neuropsychological deficits in a wide range of neurological states.

Funding The study was supported by Medical University of Gdańsk.

\section{Declarations}

Conflict of Interest The authors declare that they have no conflict of Interest.

Open Access This article is licensed under a Creative Commons Attribution 4.0 International License, which permits use, sharing, adaptation, distribution and reproduction in any medium or format, as long as you give appropriate credit to the original author(s) and the source, provide a link to the Creative Commons licence, and indicate if changes were made. The images or other third party material in this article are included in the article's Creative Commons licence, unless indicated otherwise in a credit line to the material. If material is not included in the article's Creative Commons licence and your intended use is not permitted by statutory regulation or exceeds the permitted use, you will need to obtain permission directly from the copyright holder. To view a copy of this licence, visit http://creativecommons.org/licenses/by/4.0/.

\section{References}

Annane, D., Chadda, K., Gajdos, P., Jars-Guincestre, M. C., Chevret, S., \& Raphael, J. C. (2011). Hyperbaric oxygen therapy for acute domestic carbon monoxide poisoning: Two randomized controlled trials. Intensive Care Medicine, 37(3), 486-492. https://doi.org/10.1007/s00134-010-2093-0

Baratz-Goldstein, R., Toussia-Cohen, S., Elpaz, A., Rubovitch, V., \& Pick, C. G. (2017). Immediate and delayed hyperbaric oxygen therapy as a neuroprotective treatment for traumatic brain injury in mice. Molecular and Cellular Neuroscience, 83, 74-82. https://doi.org/10.1016/j.mcn.2017.06.004

Baron, J. C. (2001). Perfusion thresholds in human cerebral ischemia: Historical perspective and therapeutic implications. Cerebrovascular Diseases, 11(SUPPL. 1), 2-8. https://doi.org/ 10.1159/000049119

Barrett, K. F., Masel, B., Patterson, J., Scheibel, R. S., Corson, K. P., \& Mader, J. T. (2004). Regional CBF in chronic stable TBI treated with hyperbaric oxygen. Undersea \& Hyperbaric Medicine : Journal of the Undersea and Hyperbaric Medical Society, Inc, 31(4), 395-406. http://www.ncbi.nlm.nih.gov/ pubmed/15686271

Bennett, M. H., Weibel, S., Wasiak, J., Schnabel, A., French, C., \& Kranke, P. (2014). Hyperbaric oxygen therapy for acute ischaemic stroke. In Cochrane Database of Systematic Reviews (Vol. 2014, Issue 11). John Wiley and Sons Ltd. https://doi. org/10.1002/14651858.CD004954.pub3

Betzen, C., White, R., Zehendner, C. M., Pietrowski, E., Bender, B., Luhmann, H. J., \& Kuhlmann, C. R. W. (2009). Oxidative stress upregulates the NMDA receptor on cerebrovascular endothelium. Free Radical Biology and Medicine, 47(8), 1212-1220. https:// doi.org/10.1016/j.freeradbiomed.2009.07.034

Bhatt, S., Puli, L., \& Patil, C. R. (2020). Role of reactive oxygen species in the progression of Alzheimer's disease. Drug Discovery Today, 00(00). https://doi.org/10.1016/j.drudis.2020.12.004

Bi, M. J., Sun, X. N., Zou, Y., Ding, X. Y., Liu, B., Zhang, Y. H., Guo, D. D., \& Li, Q. (2017). N-Butylphthalide improves cognitive function in rats after carbon monoxide poisoning. Frontiers in Pharmacology, 8(FEB), 1-12. https://doi.org/10.3389/fphar.2017.00064

Birmingham, C. M., \& Hoffman, R. S. (2011). Hyperbaric oxygen therapy for acute domestic carbon monoxide poisoning: two randomized controlled trials. Intensive Care Medicine, 37(7), 1218-1218. https://doi.org/10.1007/s00134-011-2195-3

Bitterman, N., \& Bitterman, H. (1998). L-arginine-NO pathway and CNS oxygen toxicity. Journal of Applied Physiology, 84(5), 1633-1638. https://doi.org/10.1152/jappl.1998.84.5.1633

Bliznyuk, A., Hollmann, M., \& Grossman, Y. (2020). The Mechanism of NMDA Receptor Hyperexcitation in High Pressure Helium and Hyperbaric Oxygen. Frontiers in Physiology, 11(August), 1-8. https://doi.org/10.3389/fphys.2020.01057

Boraxbekk, C. J., Salami, A., Wåhlin, A., \& Nyberg, L. (2016). Physical activity over a decade modifies age-related decline in perfusion, gray matter volume, and functional connectivity of the posterior default-mode network-A multimodal approach. NeuroImage, 131, 133-141. https://doi.org/10.1016/j.neuroimage.2015.12.010

Boussi-Gross, R., Golan, H., Fishlev, G., Bechor, Y., Volkov, O., Bergan, J., Friedman, M., Hoofien, D., Shlamkovitch, N., 
Ben-Jacob, E., \& Efrati, S. (2013). Hyperbaric oxygen therapy can improve post concussion syndrome years after mild traumatic brain injury - Randomized prospective trial. PLoS One, 8(11). https://doi.org/10.1371/journal.pone.0079995

Boussi-Gross, R., Golan, H., Volkov, O., Bechor, Y., Hoofien, D., Beeri, M. S., Ben-Jacob, E., \& Efrati, S. (2015). Improvement of memory impairments in poststroke patients by hyperbaric oxygen therapy. Neuropsychology, 29(4), 610-621. https://doi. org/10.1037/neu0000149

Brien, J. T. O., Erkinjuntti, T., Reisberg, B., Roman, G., Sawada, T., Pantoni, L., Bowler, J. V, Ballard, C., Decarli, C., Gorelick, P. B., Rockwood, K., Burns, A., Gauthier, S., \& Dekosky, S. T. (2003). Vascular cognitive impairment, Lancet Neurol, 2 (2)89-98. https://doi.org/10.1016/s1474-4422(03) 00305-3PMID: 12849265.

Bruno, A., Wagner, W., \& Orrison, W. W. (1993). Clinical outcome and brain MRI four years after carbon monoxide intoxication. Acta Neurologica Scandinavica, 87(3), 205-209. https://doi. org/10.1111/j.1600-0404.1993.tb04102.x

Burgess, N., Maguire, E. A., \& O'Keefe, J. (2002). The human hippocampus and spatial and episodic memory. In Neuron (Vol. 35, Issue 4, pp. 625-641). Cell Press. https://doi.org/10.1016/ S0896-6273(02)00830-9

Cai, F., Wang, F., Lin, F. K., Liu, C., Ma, L. Q., Liu, J., Wu, W. N., Wang, W., Wang, J. H., \& Chen, J. G. (2008). Redox modulation of long-term potentiation in the hippocampus via regulation of the glycogen synthase kinase- $3 \beta$ pathway. Free Radical Biology and Medicine, 45(7), 964-970. https://doi. org/10.1016/j.freeradbiomed.2008.06.014

Camporesi, E. M. (2014). Side effects of hyperbaric oxygen therapy. Undersea and Hyperbaric Medicine, 41(3), 253-257.

Chang, C. C., Chang, W. N., Lui, C. C., Wang, J. J., Chen, C. F., Lee, Y. C., Chen, S. S., Lin, Y. T., Huang, C. W., Chen, C., C. C., Chang, M. P., Chang, W., \& Chen, S. (2010a). Longitudinal study of carbon monoxide intoxication by diffusion tensor imaging with neurospsychiatric correlation. Journal of Psychiatry \& Neuroscience, 35(2). https://doi.org/10.1503/jpn.090057

Chang, D. C., Lee, J. T., Lo, C. P., Fan, Y. M., Huang, K. L., Kang, B. H., Hsieh, H. L., \& Chen, S. Y. (2010b). Hyperbaric oxygen ameliorates delayed neuropsychiatric syndrome of carbon monoxide poisoning. Undersea \& Hyperbaric Medicine, 37(1), 23-33. http://www.ncbi.nlm.nih.gov/pubmed/20369650

Chen, X., Duan, X. S., Xu, L. J., Zhao, J. J., She, Z. F., Chen, W. W., Zheng, Z. J., \& Jiang, G. D. (2014). Interleukin-10 mediates the neuroprotection of hyperbaric oxygen therapy against traumatic brain injury in mice. Neuroscience, 266, 235-243. https://doi. org/10.1016/j.neuroscience.2013.11.036

Chen, Z., Ni, P., Lin, Y., Xiao, H., Chen, J., Qian, G., Ye, Y., Xu, S., Wang, J., \& Yang, X. (2010). Visual pathway lesion and its development during hyperbaric oxygen treatment: A bold-fMRI and DTI study. Journal of Magnetic Resonance Imaging, 31(5), 1054-1060. https://doi.org/10.1002/jmri.22142

Chiu, W. T., \& LaPorte, R. E. (1993). Global Spine and Head Injury Prevention (SHIP) Project. The Journal of Trauma, 35(6), 969-970. http://www.ncbi.nlm.nih.gov/pubmed/8264006

Choi, S. (1983). Delayed Neurologic Sequelae in Carbon Monoxide Intoxication. Archives of Neurology, 40(7), 433-435. https:// doi.org/10.1001/archneur.1983.04050070063016

Churchill, S., Scott Miller, R., Deru, K., Wilson, S. H., \& Weaver, L. K. (2016). Simple and procedural reaction time for mild traumatic brain injury in a hyperbaric oxygen clinical trial. Military Medicine, 181(5), 40-44. https://doi.org/10.7205/MILMED-D-15-00148

Churchill, S., Weaver, L. K., Deru, K., Russo, A. A., Handrahan, D., Orrison, W. W., Foley, J. F., \& Elwell, H. A. (2013). A prospective trial of hyperbaric oxygen for chronic sequelae after brain injury (HYBOBI). Undersea and Hyperbaric Medicine, 40(2), 165-193.
Cifu, D. X., Hart, B. B., West, S. L., Walker, W., \& Carne, W. (2014). The effect of hyperbaric oxygen on persistent postconcussion symptoms. Journal of Head Trauma Rehabilitation, 29(1), 11-20. https://doi.org/10.1097/HTR.0b013e3182a6aaf0

Clark, K. B., Naritoku, D. K., Smith, D. C., Browning, R. A., \& Jensen, R. A. (1999). Enhanced recognition memory following vagus nerve stimulation in human subjects. Nature Neuroscience, 2(1), 94-98. https://doi.org/10.1038/4600

Ferri, C. P., Prince, M., Brayne, C., Brodaty, H., Fratiglioni, L., Ganguli, M., Hall, K., Hasegawa, K., Hendrie, H., Huang, Y., Jorm, A., Mathers, C., Menezes, P. R., Rimmer, E., \& Scazufca, M. (2005). Global prevalence of dementia: a Delphi consensus study. Lancet, 366, 2112-2117.

Daly, S., Thorpe, M., Rockswold, S., Hubbard, M., Bergman, T., Samadani, U., \& Rockswold, G. (2018). Hyperbaric Oxygen Therapy in the Treatment of Acute Severe Traumatic Brain Injury: A Systematic Review. In Journal of Neurotrauma (Vol. 35, Issue 4, pp. 623-629). Mary Ann Liebert Inc. https://doi. org/10.1089/neu.2017.5225

Deng, Z., Chen, W., Jin, J., Zhao, J., \& Xu, H. (2018). The neuroprotection effect of oxygen therapy: A systematic review and meta-analysis. In Nigerian Journal of Clinical Practice (Vol. 21, Issue 4, pp. 401-416). Medknow Publications. https://doi. org/10.4103/njcp.njcp_315_16

Di Piero, V., Cappagli, M., Pastena, L., Faralli, F., Mainardi, G., Di Stani, F., Bruti, G., Coli, A., Lenzi, G. L., \& Gagliardi, R. (2002). Cerebral effects of hyperbaric oxygen breathing: A CBF SPECT study on professional divers. European Journal of Neurology, 9(4), 419-421. https://doi.org/10.1046/j.1468-1331.2002.00436.x

Edwards, A. E., \& Hart, G. M. (1974). Hyperbaric Oxygenation and the Cognitive Functioning of the Aged. Journal of the American Geriatrics Society, 22(8), 376-379. https://doi. org/10.1111/j.1532-5415.1974.tb04845.x

Efrati, S., \& Ben-Jacob, E. (2014a). Reflections on the neurotherapeutic effects of hyperbaric oxygen. Expert Review of Neurotherapeutics, 14(3), 233-236. https://doi.org/10.1586/14737175.2014.884928

Efrati, S., \& Ben-Jacob, E. (2014b). Reflections on the neurotherapeutic effects of hyperbaric oxygen. In Expert Review of Neurotherapeutics (Vol. 14, Issue 3, pp. 233-236). https://doi. org/10.1586/14737175.2014.884928

Efrati, S., Fishlev, G., Bechor, Y., Volkov, O., Bergan, J., Kliakhandler, K., Kamiager, I., Gal, N., Friedman, M., Ben-Jacob, E., \& Golan, H. (2013). Hyperbaric Oxygen Induces Late Neuroplasticity in Post Stroke Patients - Randomized, Prospective Trial. PLoS One, 8(1). https://doi.org/10.1371/journal.pone.0053716

Erkinjuntti, T., Román, G., Gauthier, S., Feldman, H., \& Rockwood, K. (2004). Emerging Therapies for Vascular Dementia and Vascular Cognitive Impairment. Stroke, 35(4), 1010-1017. https://doi.org/10.1161/01.STR.0000120731.88236.33

Ernst, A., \& Zibrak, J. D. (1998). Carbon Monoxide Poisoning. New England Journal of Medicine, 339(22), 1603-1608. https://doi. org/10.1056/NEJM199811263392206

Fischer, B., Palkovic, S., Holling, M., Wolfer, J., \& Wassmann, H. (2010). Rationale of Hyperbaric Oxygenation in Cerebral Vascular Insult. Current Vascular Pharmacology, 8(1), 35-43. https://doi.org/10.2174/157016110790226598

Francis, A., \& Baynosa, R. (2017). Ischaemia-reperfusion injury and hyperbaric oxygen pathways: A review of cellular mechanisms. In Diving and Hyperbaric Medicine (Vol. 47, Issue 2, pp. 110-117). South Pacific Medicine Underwater Society and the European Underwater and Baromedical Society. https://doi. org/10.28920/dhm47.2.110-117

Freiberger, J. J., Derrick, B. J., Natoli, M. J., Akushevich, I., Schinazi, E. A., Parker, C., Stolp, B. W., Bennett, P. B., Vann, R. D., Dunworth, S. A. S., \& Moon, R. E. (2016). Assessment of the interaction of hyperbaric $\mathrm{N} 2, \mathrm{CO} 2$, and $\mathrm{O} 2$ on psychomotor performance in 
divers. Journal of Applied Physiology, 121(4), 953-964. https:// doi.org/10.1152/japplphysiol.00534.2016

Gale, S. D., \& Hopkins, R. O. (2004). Effects of hypoxia on the rain: Neuroimaging and neuropsychological findings following carbon monoxide poisoning and obstructive sleep apnea. Journal of the International Neuropsychological Society, 10(1), 60-71. https://doi.org/10.1017/S1355617704101082

Garcia, A. J., Putnam, R. W., \& Dean, J. B. (2010a). Hyperbaric hyperoxia and normobaric reoxygenation increase excitability and activate oxygen-induced potentiation in CA1 hippocampal neurons. Journal of Applied Physiology, 109(3), 804-819. https://doi.org/10.1152/japplphysiol.91429.2008

Garcia, A. J., Putnam, R. W., \& Dean, J. B. (2010b). Hyperoxic stimulation of synchronous orthodromic activity and induction of neural plasticity does not require changes in excitatory synaptic transmission. Journal of Applied Physiology, 109(3), 820-829. https://doi.org/10.1152/japplphysiol.91430.2008

Ghacibeh, G. A., Shenker, J. I., Shenal, B., Uthman, B. M., \& Heilman, K. M. (2006). The influence of vagus nerve stimulation on memory. Cognitive and Behavioral Neurology, 19(3), 119-122. https://doi. org/10.1097/01.wnn.0000213908.34278.7d

Godefroy, O., \& Bogousslavsky, J. (2007). The behavioral and cognitive neurology of stroke. The Behavioral and Cognitive Neurology of Stroke. https://doi.org/10.1017/CBO9780511544880

Golden, Z., Golden, C. J., \& Neubauer, R. A. (2006). Improving neuropsychological function after chronic brain injury with hyperbaric oxygen. Disability and Rehabilitation, 28(22), 1379-1386. https://doi.org/10.1080/09638280600638364

Golden, Z. L., Neubauer, R., Golden, C. J., Greene, L., Marsh, J., \& Mleko, A. (2002). Improvement in cerebral metabolism in chronic brain injury after Hyperbaric Oxygen Therapy. International Journal of Neuroscience, 112(2), 119-131. https:// doi.org/10.1080/00207450212027

Goldfarb, A. I., Hochstadt, N. J., Jacobson, J. H., \& Weinstein, E. A. (1972). Hyperbaric oxygen treatment of organic mental syndrome in aged persons. Journal of Gerontology, 27(2), 212-217. https://doi.org/10.1093/geronj/27.2.212

Goldstein, M. (2008). Carbon Monoxide Poisoning. Journal of Emergency Nursing, 34(6), 538-542. https://doi.org/10.1016/j. jen.2007.11.014

Gong, L. W., Gao, T. M., Huang, H., \& Tong, Z. (2000). Redox modulation of large conductance calcium-activated potassium channels in CA1 pyramidal neurons from adult rat hippocampus. Neuroscience Letters, 286(3), 191-194. https://doi. org/10.1016/S0304-3940(00)01121-6

Grim, P. S. (1990). Hyperbaric Oxygen Therapy. JAMA: The Journal of the American Medical Association, 263(16), 2216. https:// doi.org/10.1001/jama.1990.03440160078042

Guiney, H., Lucas, S. J. E., Cotter, J. D., \& Machado, L. (2019). Investigating links between habitual physical activity, cerebrovascular function, and cognitive control in healthy older adults. Neuropsychologia, 125(July 2018), 62-69. https://doi. org/10.1016/j.neuropsychologia.2019.01.011

Hadanny, A., Golan, H., Fishlev, G., Bechor, Y., Volkov, O., Suzin, G., Ben-Jacob, E., \& Efrati, S. (2015). Hyperbaric oxygen can induce neuroplasticity and improve cognitive functions of patients suffering from anoxic brain damage. Restorative Neurology and Neuroscience, 33(4), 471-486. https://doi.org/ 10.3233/RNN-150517

Hadanny, A., Meir, O., Bechor, Y., Fishlev, G., Bergan, J., \& Efrati, S. (2016). Seizures during hyperbaric oxygen therapy: Retrospective analysis of 62,614 treatment sessions. Undersea and Hyperbaric Medicine, 43(1), 21-28.

Hadanny, A., Meir, O., Bechor, Y., Fishlev, G., Bergan, J., \& Efrati, S. (2016). The safety of hyperbaric oxygen treatment
- Retrospective analysis in 2,334 patients. Undersea and Hyperbaric Medicine, 43(2), 113-122.

Hadanny, A., Abbott, S., Suzin, G., Bechor, Y., \& Efrati, S. (2018). Effect of hyperbaric oxygen therapy on chronic neurocognitive deficits of posttraumatic brain injury patients: Retrospective analysis. British Medical Journal Open, 8(9), 1-9. https://doi. org/10.1136/bmjopen-2018-023387

Hadanny, A., \& Efrati, S. (2016). The efficacy and safety of hyperbaric oxygen therapy in traumatic brain injury. In Expert Review of Neurotherapeutics (Vol. 16, Issue 4, pp. 359-360). Taylor and Francis Ltd. https://doi.org/10.1586/14737175.2016.1157018

Hadanny, A., Rittblat, M., Bitterman, M., May-Raz, I., Suzin, G., Boussi-Gross, R., Zemel, Y., Bechor, Y., Catalogna, M., \& Efrati, S. (2020). Hyperbaric oxygen therapy improves neurocognitive functions of post-stroke patients-a retrospective analysis. Restorative Neurology and Neuroscience, 38(1), 93-107. https://doi. org/10.3233/RNN-190959

Hampson, N. B., Piantadosi, C. A., Thom, S. R., \& Weaver, L. K. (2013). Practice recommendations in the diagnosis, management, and prevention of carbon monoxide poisoning. Diving and Hyperbaric Medicine, 43(3), 172. https://doi.org/10.1164/ rccm.201207-1284CI

Harch, P. G., Andrews, S. R., Fogarty, E. F., Amen, D., Pezzullo, J. C., Lucarini, J., Aubrey, C., Taylor, D. V., Staab, P. K., \& Van Meter, K. W. (2012). A phase i study of low-pressure hyperbaric oxygen therapy for blast-induced post-concussion syndrome and post-traumatic stress disorder. Journal of Neurotrauma, 29(1), 168-185. https://doi.org/10.1089/neu.2011.1895

Harch, P. G., Kriedt, C., Van Meter, K. W., \& Sutherland, R. J. (2007). Hyperbaric oxygen therapy improves spatial learning and memory in a rat model of chronic traumatic brain injury. Brain Research, 1174(1), 120-129. https://doi.org/10.1016/j.brainres.2007.06.105

Hardy, P., Collet, J. P., Goldberg, J., Vanasse, M., Lambert, J., Marois, P., Amar, M., Montgomery, D. L., Lecomte, J. M., Johnston, K. M., \& Lassonde, M. (2002). Neuropsychological effects of hyperbaric oxygen therapy in cerebral palsy. Developmental Medicine and Child Neurology, 44(7), 436-446. https://doi. org/10.1017/S0012162201002341

Hart, G. B., \& Strauss, M. B. (2003). Hyperbaric Oxygen Therapy. Stroke, 34(9). https://doi.org/10.1161/01.STR.0000087115.91721.6E

Hart, G. B., \& Thompson, R. E. (1971). The treatment of cerebral ischemia with $\mathrm{H} /$ perbaric Oxygen (OHP). Stroke, 2(3), 247-250. https://doi.org/10.1161/01.STR.2.3.247

Helms, A. K., Whelan, H. T., \& Torbey, M. T. (2005). Hyperbaric Oxygen Therapy of Cerebral Ischemia. Cerebrovascular Diseases, 20(6), 417-426. https://doi.org/10.1159/000088979

Henke, K., Kroll, N. E., Behniea, H., Amaral, D. G., Miller, M. B., Rafal, R., \& Gazzaniga, M. S. (1999). Memory lost and regained following bilateral hippocampal damage. Journal of Cognitive Neuroscience, 11(6), 682-697. http://www.ncbi.nlm.nih.gov/ pubmed/10601749

Hopkins, R. O., Weaver, L. K., Valentine, K. J., Mower, C., Churchill, S., \& Carlquist, J. (2007). Apolipoprotein E genotype and response of carbon monoxide poisoning to hyperbaric oxygen treatment. American Journal of Respiratory and Critical Care Medicine, 176(10), 1001-1006. https://doi.org/10.1164/rccm.200702-290OC

Hsu, S. M., Tseng, C. H., Hsieh, C. H., \& Hsieh, C. W. (2020). Slowpaced inspiration regularizes alpha phase dynamics in the human brain. Journal of Neurophysiology, 123(1), 289-299. https://doi. org/10.1152/JN.00624.2019

Hu, D., Klann, E., \& Thiels, E. (2007). Superoxide dismutase and hippocampal function: Age and isozyme matter. In Antioxidants and Redox Signaling (Vol. 9, Issue 2, pp. 201-210). Antioxid Redox Signal. https://doi.org/10.1089/ars.2007.9.201

Huddleston, A. T., Tang, W., Takeshima, H., Hamilton, S. L., \& Klann, E. (2008). Superoxide-induced potentiation in the hippocampus 
requires activation of ryanodine receptor type 3 and ERK. Journal of Neurophysiology, 99(3), 1565-1571. https://doi.org/10. 1152/jn.00659.2007

Jackson, P. A., Pialoux, V., Corbett, D., Drogos, L., Erickson, K. I., Eskes, G. A., \& Poulin, M. J. (2016). Promoting brain health through exercise and diet in older adults: a physiological perspective. Journal of Physiology, 594(16), 4485-4498. https://doi.org/ $10.1113 / \mathrm{JP} 271270$

Jacobs, E. A., Alvis, H. J., \& Small, S. M. (1972). Hyperoxygenation: a central nervous system activator? Journal of Geriatric Psychiatry, 5(2), 107-136. http://www.ncbi.nlm.nih.gov/pubmed/4584068

Jacobs, E. A., Winter, P. M., Alvis, H. J., \& Small, S. M. (1969). Hyperoxygenation effect on cognitive functioning in the aged. The New England Journal of Medicine, 281(14), 753-757. https://doi.org/ 10.1056/NEJM196910022811402

Jain, K. K. (2017). Textbook of Hyperbaric Medicine. https://doi.org/ 10.1007/978-3-319-47140-2

Kalaria, R. N., Maestre, G. E., Arizaga, R., Friedland, R. P., Galasko, D., Hall, K., Luchsinger, J. A., Ogunniyi, A., Perry, E. K., Potocnik, F., Prince, M., Stewart, R., Wimo, A., Zhang, Z. X., \& Antuono, P. (2008). Alzheimer's disease and vascular dementia in developing countries: prevalence, management, and risk factors. In The Lancet Neurology (Vol. 7, Issue 9, pp. 812-826). Lancet Publishing Group. https://doi.org/10.1016/S1474-4422(08)70169-8

Kandlur, A., Satyamoorthy, K., \& Gangadharan, G. (2020). Oxidative Stress in Cognitive and Epigenetic Aging: A Retrospective Glance. Frontiers in Molecular Neuroscience, 13(March). https://doi.org/ 10.3389/fnmol.2020.00041

Kemmerling, U., Muñoz, P., Müller, M., Sánchez, G., Aylwin, M. L., Klann, E., Carrasco, M. A., \& Hidalgo, C. (2007). Calcium release by ryanodine receptors mediates hydrogen peroxideinduced activation of ERK and CREB phosphorylation in N2a cells and hippocampal neurons. Cell Calcium, 41(5), 491-502. https://doi.org/10.1016/j.ceca.2006.10.001

Kety, S. S., \& Schmidt, C. F. (1948). the Effects of Altered Arterial Tensions of Carbon Dioxide and Oxygen on Cerebral Blood Flow and Cerebral Oxygen Consumption of Normal Young Men. The Journal of Clinical Investigation, 27(4), 484-492. https://doi. org/10.1172/JCI101995

Kot, J., Winklewski, P. J., Sicko, Z., \& Tkachenko, Y. (2015). Effect of oxygen on neuronal excitability measured by critical flicker fusion frequency is dose dependent. Journal of Clinical and Experimental Neuropsychology, 37(3), 276-284. https://doi.org/ 10.1080/13803395.2015.1007118

Kraitsy, K., Uecal, M., Grossauer, S., Bruckmann, L., Pfleger, F., Ropele, S., Fazekas, F., Gruenbacher, G., Patz, S., Absenger, M., Porubsky, C., Smolle-Juettner, F., Tezer, I., Molcanyi, M., Fasching, U., \& Schaefer, U. (2014). Repetitive long-term Hyperbaric Oxygen Treatment,(HBOT)administered after experimental traumatic brain injury in rats induces significant remyelination and a recovery of sensorimotor function. PLoS One, 9(5). https://doi.org/10.1371/journal.pone.0097750

Kushner, D. (1998). Mild traumatic brain injury: Toward understanding manifestations and treatment. In Archives of Internal Medicine (Vol. 158, Issue 15, pp. 1617-1624). https://doi.org/10.1001/ archinte.158.15.1617

Kuźma, E., Lourida, I., Moore, S. F., Levine, D. A., Ukoumunne, O. C., \& Llewellyn, D. J. (2018). Stroke and dementia risk: A systematic review and meta-analysis. Alzheimer's and Dementia, 14(11), 1416-1426. https://doi.org/10.1016/j.jalz.2018.06.3061

Leach, R. M., Rees, P. J., \& Wilmshurst, P. (1998). ABC of oxygen: Hyperbaric oxygen therapy. In British Medical Journal (Vol. 317, Issue 7166, pp. 1140-1143). https://doi.org/10.1136/bmj. 317.7166 .1140

Lee, M. S., Kim, J. S., Chung, T. S., \& Suh, J. H. (1988). Measurements of cerebral blood flow in delayed carbon monoxide sequelae using xenon inhalation CT scan. Yonsei Medical Journal, 29(2), 185-192. https://doi.org/10.3349/ymj.1988.29.2.185

Lee, Y. S., Chio, C. C., Chang, C. P., Wang, L. C., Chiang, P. M., Niu, K. C., \& Tsai, K. J. (2013). Long course hyperbaric oxygen stimulates neurogenesis and attenuates inflammation after ischemic stroke. Mediators of Inflammation, 2013, 512978. https://doi.org/10.1155/2013/512978

Levin, H. S. (1990). Memory deficit after closed-head injury. Journal of Clinical and Experimental Neuropsychology, 12(1), 129-153. https://doi.org/10.1080/01688639008400960

Liao, S. C., Mao, Y. C., Yang, K. J., Wang, K. C., Wu, L. Y., \& Yang, C. C. (2019). Targeting optimal time for hyperbaric oxygen therapy following carbon monoxide poisoning for prevention of delayed neuropsychiatric sequelae: A retrospective study. Journal of the Neurological Sciences, 396, 187-192. https://doi. org/10.1016/j.jns.2018.11.025

Lin, K. C., Niu, K. C., Tsai, K. J., Kuo, J. R., Wang, L. C., Chio, C. C., \& Chang, C. P. (2012). Attenuating inflammation but stimulating both angiogenesis and neurogenesis using hyperbaric oxygen in rats with traumatic brain injury. Journal of Trauma and Acute Care Surgery, 72(3), 650-659. https://doi.org/10.1097/TA. 0b013e31823c575f

Liu, S., Shen, G. Y., Deng, S. K., Wang, X. B., Wu, Q. F., \& Guo, A. S. (2013). Hyperbaric oxygen therapy improves cognitive functioning after brain injury. Neural Regeneration Research, 8(35), 3334-3343. https://doi.org/10.3969/j. issn.1673-5374.2013.35.008

Liu, W. C., Yang, S. N., Wu, C. W. J., Chen, L. W., \& Chan, J. Y. H. (2016). Hyperbaric oxygen therapy alleviates carbon monoxide poisoning-induced delayed memory impairment by preserving brain-derived neurotrophic factor-dependent hippocampal neurogenesis. Critical Care Medicine, 44(1), e25-e39. https://doi. org/10.1097/CCM.0000000000001299

Lo, C. P., Chen, S. Y., Chou, M. C., Wang, C. Y., Lee, K. W., Hsueh, C. J., Chen, C. Y., Huang, K. L., \& Huang, G. S. (2007). Diffusion-tensor MR imaging for evaluation of the efficacy of hyperbaric oxygen therapy in patients with delayed neuropsychiatric syndrome caused by carbon monoxide inhalation. European Journal of Neurology, 14(7), 777-782. https://doi.org/10.1111/j.1468-1331.2007.01854.x

Lo, E. H., Dalkara, T., \& Moskowitz, M. A. (2003). Neurological diseases: Mechanisms, challenges and opportunities in stroke. Nature Reviews Neuroscience, 4(5), 399-414. https://doi. org/10.1038/nrn1106

Lodato, R. F., \& Jubran, A. (1993). Response time, autonomic mediation, and reversibility of hyperoxic bradycardia in conscious dogs. Journal of Applied Physiology, 74(2), 634-642. https:// doi.org/10.1152/jappl.1993.74.2.634

Mannaioni, P. F., Vannacci, A., \& Masini, E. (2006). Carbon monoxide: The bad and the good side of the coin, from neuronal death to anti-inflammatory activity. In Inflammation Research (Vol. 55, Issue 7, pp. 261-273). Inflamm Res. https://doi.org/10.1007/ s00011-006-0084-y

Maric, V., Ramanathan, D., \& Mishra, J. (2020). Respiratory regulation $\&$ interactions with neuro-cognitive circuitry. Neuroscience and Biobehavioral Reviews, 112(February 2019), 95-106. https://doi. org/10.1016/j.neubiorev.2020.02.001

Massaad, C. A., \& Klann, E. (2011). Reactive oxygen species in the regulation of synaptic plasticity and memory. Antioxidants and Redox Signaling, 14(10), 2013-2054. https://doi.org/10.1089/ ars. 2010.3208

Mathieu, D., Nolf, M., Durocher, A., Saulnier, F., Frimat, P., Furon, D., \& Wattel, F. (1985). Acute carbon monoxide poisoning risk of late sequelae and treatment by hyperbaric oxygen. Clinical Toxicology, 23(4-6), 315-324. https://doi.org/10.3109/15563658508990639 Mathieu, D. (2006a). Handbook on hyperbaric medicine. Springer. 
Mathieu, D. (Ed.). (2006b). Handbook on Hyperbaric Medicine. Springer Netherlands. https://doi.org/10.1007/1-4020-4448-8

Mathieu, D., Marroni, A., \& Kot, J. (2017). Tenth european consensus conference on hyperbaric medicine: Recommendations for accepted and non-accepted clinical indications and practice of hyperbaric oxygen treatment. Diving and Hyperbaric Medicine, 47(1), 24-31. https://doi.org/10.28920/dhm47.1.24-32

McCauley, R. D., Fewtrell, J., Duncan, A. J., Jenner, C., Jenner, M.-N., Penrose, J. D., et al. (2000). Marineseismic surveys - a study of environmental implications. The APPEA Journal, 40(1), 692. https://doi.org/10.1071/aj99048.

Medana, I. M., \& Esiri, M. M. (2003). Axonal damage: A key predictor of outcome in human CNS diseases. In Brain (Vol. 126, Issue 3, pp. 515-530). https://doi.org/10.1093/brain/awg061

Micarelli, A., Jacobsson, H., Larsson, S. A., Jonsson, C., \& Pagani, M. (2013). Neurobiological insight into hyperbaric hyperoxia. Acta Physiologica, 209(1), 69-76. https://doi.org/10.1111/apha.12116

Moher, D., Liberati, A., Tetzlaff, J., Altman, D. G., Altman, D., Antes, G., Atkins, D., Barbour, V., Barrowman, N., Berlin, J. A., Clark, J., Clarke, M., Cook, D., D’Amico, R., Deeks, J. J., Devereaux, P. J., Dickersin, K., Egger, M., Ernst, E., ... Tugwell, P. (2009). Preferred reporting items for systematic reviews and meta-analyses: The PRISMA statement. In PLoS Medicine (Vol. 6, Issue 7). PLoS Med. https://doi.org/10.1371/journal.pmed.1000097

Moon, R. E. (2019). Hyperbaric Oxygen Therapy INDICATIONS 14 TH EDITION.

Moorhouse, P., \& Rockwood, K. (2008). Vascular cognitive impairment: current concepts and clinical developments. In The Lancet Neurology (Vol. 7, Issue 3, pp. 246-255). https://doi.org/10.1016/ S1474-4422(08)70040-1

Mor, A., \& Grossman, Y. (2010). The efficacy of physiological and pharmacological $\mathrm{N}$-methyl-d-aspartate receptor block is greatly reduced under hyperbaric conditions. Neuroscience, 169(1), 1-7. https://doi.org/10.1016/j.neuroscience.2010.05.009

Mor, A., \& Grossman, Y. (2007). High pressure modulation of NMDA receptor dependent excitability. European Journal of Neuroscience, 25(7), 2045-2052. https://doi.org/10.1111/j.1460-9568.2007.05479.x

Mu, J., Krafft, P. R., \& Zhang, J. H. (2011). Hyperbaric oxygen therapy promotes neurogenesis: Where do we stand? In Medical Gas Research (Vol. 1, Issue 1). BioMed Central Ltd. https://doi.org/ 10.1186/2045-9912-1-14

Mundy, M. E., Downing, P. E., Dwyer, D. M., Honey, R. C., \& Graham, K. S. (2013). A critical role for the hippocampus and perirhinal cortex in perceptual learning of scenes and faces: Complementary findings from amnesia and fMRI. Journal of Neuroscience, 33(25), 10490-10502. https://doi.org/10.1523/JNEUROSCI.2958-12.2013

Mychaskiw, G., \& Stephens, P. L. (2013). Hyperbaric Oxygen, Mild Traumatic Brain Injury, and Study Design: An Elusive Target. Journal of Neurotrauma, 30(19), 1681-1682. https://doi. org/10.1089/neu.2012.2805

Neubauer, R. A., \& James, P. (1998). Cerebral oxygenation and the recoverable brain. Neurological Research, 20(SUPPL. 1). https:// doi.org/10.1080/01616412.1998.11740606

Ogoh, S., \& Tarumi, T. (2019). Cerebral blood flow regulation and cognitive function: a role of arterial baroreflex function. Journal of Physiological Sciences, 69(6), 813-823. https://doi.org/10.1007/ s12576-019-00704-6

Omae, T., Ibayashi, S., Kusuda, K., Nakamura, H., Yagi, H., \& Fujishima, M. (1998). Effects of high atmospheric pressure and oxygen on middle cerebral blood flow velocity in humans measured by transcranial Doppler. Stroke, 29(1), 94-97. https://doi. org/10.1161/01.STR.29.1.94

Oriani, G., Marroni, A., \& Wattel, F. (1996). Handbook on Hyperbaric Medicine. Springer.

Oury, T. D., Ho, Y. S., Piantadosi, C. A., \& Crapo, J. D. (1992). Extracellular superoxide dismutase, nitric oxide, and central nervous system $\mathrm{O} 2$ toxicity. Proceedings of the National Academy of Sciences of the United States of America, 89(20), 9715-9719. https:// doi.org/10.1073/pnas.89.20.9715

Park, Y. M., \& Kim, Y. S. (2014). Secondary mania in a patient with delayed anoxic encephalopathy after carbon monoxide intoxication caused by a suicide attempt. General Hospital Psychiatry, 36(1), 125.e3-125.e4. https://doi.org/10.1016/j.genhosppsych.2013.07.003

Parkinson, J., \& Registrar, M. (1871). the Rate of the Pulse in Health . By.

Potter, S., Leigh, E., Wade, D., \& Fleminger, S. (2006). The Rivermead Post Concussion Symptoms Questionnaire: A confirmatory factor analysis. Journal of Neurology, 253(12), 1603-1614. https:// doi.org/10.1007/s00415-006-0275-z

Prior, H., Schwegler, H., \& Dücker, G. (1997). Dissociation of spatial reference memory, spatial working memory, and hippocampal mossy fiber distribution in two rat strains differing in emotionality. Behavioural Brain Research, 87(2), 183-194. https://doi.org/ 10.1016/S0166-4328(97)02282-1

Prockop, L. D., \& Chichkova, R. I. (2007). Carbon monoxide intoxication: An updated review. Journal of the Neurological Sciences, 262(1-2), 122-130. https://doi.org/10.1016/j.jns.2007.06.037

Rajeswaran, J. (2013). Neuropsychological Rehabilitation Principles and Applications Edited. Journal of Chemical Information and Modeling. https://doi.org/10.1017/CBO9781107415324.004

Raskin, A., Gershon, S., Crook, T. H., Sathananthan, G., \& Ferris, S. (1978). The Effects of Hyperbaric and Normobaric Oxygen on Cognitive Impairment in the Elderly. Archives of General Psychiatry, 35(1), 50-56. https://doi.org/10.1001/archpsyc.1978.01770250052005

Ricker, J. H., \& Callahan, C. D. (2000). International Handbook of Neuropsychological Rehabilitation. In Journal of Head Trauma Rehabilitation (Vol. 15, Issue 3). https://doi. org/10.1097/00001199-200006000-00012

Robinson, R. G. (2006). The clinical neuropsychiatry of stroke: Second edition. In The Clinical Neuropsychiatry of Stroke. https://doi. org/10.1017/CBO9780511544231

Rockswold, S. B., Rockswold, G. L., Zaun, D. A., \& Liu, J. (2013). A prospective, randomized Phase II clinical trial to evaluate the effect of combined hyperbaric and normobaric hyperoxia on cerebral metabolism, intracranial pressure, oxygen toxicity, and clinical outcome in severe traumatic brain injury. Journal of Neurosurgery, 118(6), 1317-1328. https://doi. org/10.3171/2013.2.JNS121468

Rockswold, S. B., Rockswold, G. L., Zaun, D. A., Zhang, X., Cerra, C. E., Bergman, T. A., \& Liu, J. (2010). A prospective, randomized clinical trial to compare the effect of hyperbaric to normobaric hyperoxia on cerebral metabolism, intracranial pressure, and oxygen toxicity in severe traumatic brain injury: Clinical article. Journal of Neurosurgery, 112(5), 1080-1094. https://doi. org/10.3171/2009.7.JNS09363

Rosario, E. R., Kaplan, S. E., Khonsari, S., Vazquez, G., Solanki, N., Lane, M., Brownell, H., \& Rosenberg, S. S. (2018). The Effect of Hyperbaric Oxygen Therapy on Functional Impairments Caused by Ischemic Stroke. Neurology Research International, 2018, 12-15. https://doi.org/10.1155/2018/3172679

Rossignol, D. A., Rossignol, L. W., Jill, S. J., Melnyk, S., \& Mumper, E. (2007). The effects of hyperbaric oxygen therapy on oxidative stress, inflammation, and symptoms in children with autism: An open-label pilot study. BMC Pediatrics, 7, 1-13. https://doi. org/10.1186/1471-2431-7-36

Sánchez, E. C. (2013). Mechanisms of action of hyperbaric oxygenation in stroke: A review. In Critical Care Nursing Quarterly (Vol. 36, Issue 3, pp. 290-298). https://doi.org/10.1097/CNQ.0b013e318294e9e3

Sarno, J. E., Rusk, H. A., Diller, L., \& Sarno, M. T. (1972a). The effect of hyperbaric oxygen on the mental and verbal ability of stroke patients. Stroke, 3(1), 10-15. https://doi.org/10.1161/01. STR.3.1.10 
Sarno, M. T., Sarno, J. E., \& Diller, L. (1972b). The effect of hyperbaric oxygen on communication function in adults with aphasia secondary to stroke. Journal of Speech and Hearing Research, 15(1), 42-48. https://doi.org/10.1044/jshr.1501.42

Scheinkestel, C. D., Bailey, M., Myles, P. S., Jones, K., Cooper, D. J., Millar, I. L., \& Tuxen, D. V. (1999). Hyperbaric or normobaric oxygen for acute carbon monoxide poisoning: a randomised controlled clinical trial. The Medical Journal of Australia, 170(5), 203-210. http://www.ncbi.nlm.nih.gov/pubmed/10092916

Shandley, S., Wolf, E. G., Schubert-Kabban, C. M., Baugh, L. M., Richards, M. F., Prye, J., Arizpe, H. M., \& Kalns, J. (2017). Increased circulating stem cells and better cognitive performance in traumatic brain injury subjects following hyperbaric oxygen therapy. Undersea and Hyperbaric Medicine, 44(3), 257-269. https://doi.org/10.22462/5.6.2017.6

Shapira, R., Solomon, B., Efrati, S., Frenkel, D., \& Ashery, U. (2018). Hyperbaric oxygen therapy ameliorates pathophysiology of $3 \times \mathrm{Tg}-\mathrm{AD}$ mouse model by attenuating neuroinflammation. Neurobiology of Aging, 62, 105-119. https://doi.org/10.1016/j. neurobiolaging.2017.10.007

Shetty, P. K., Huang, F. L., \& Huang, K. P. (2008). Ischemia-elicited oxidative modulation of $\mathrm{Ca} 2+/$ calmodulin- dependent protein kinase II. Journal of Biological Chemistry, 283(9), 5389-5401. https://doi.org/10.1074/jbc.M708479200

Shi, X., Tang, Z., Xiong, B., Bao, J., Sun, D., Zhang, Y., \& Yao, Y. (2003). Cerebral perfusion SPECT imaging for assessment of the effect of hyperbaric oxygen therapy on patients with postbrain injury neural status. Chinese Journal of Traumatology = Zhonghua Chuang Shang Za Zhi, 6(6), 346-349. http://www.ncbi.nlm. nih.gov/pubmed/14642054

Singhal, A. B. (2007). A review of oxygen therapy in ischemic stroke. In Neurological Research (Vol. 29, Issue 2, pp. 173183). https://doi.org/10.1179/016164107X181815

Smith, R., Thayer, J. F., Khalsa, S. S., \& Lane, R. D. (2017). The hierarchical basis of neurovisceral integration. Neuroscience and Biobehavioral Reviews, 75, 274-296. https://doi.org/10.1016/j. neubiorev.2017.02.003

Sohlberg, M. M., \& Mateer, C. A. (2001). Cognitive rehabilitation: An integrative neuropsychological approach. - PsycNET (G. Press. (Ed.)). https://psycnet.apa.org/record/2001-01866-000

Sohlberg, M. M., \& Mateer, C. A. (2001). Improving attention and managing attentional problems. Adapting rehabilitation techniques to adults with ADD. Annals of the New York Academy of Sciences, 931, 359-375. https://doi.org/10.1111/j.1749-6632.2001.tb05790.x

Sönmez, B. M., İşcanl, M. D., Parlak, S., Doğan, Y., Ulubay, H. G., \& Temel, E. (2018). Delayed neurologic sequelae of carbon monoxide intoxication. Turkish Journal of Emergency Medicine, 18(4), 167-169. https://doi.org/10.1016/j.tjem.2018.04.002

Sorrentino, G., Migliaccio, R., \& Bonavita, V. (2008). Treatment of vascular dementia: The route of prevention. In European Neurology (Vol. 60, Issue 5, pp. 217-223). https://doi. org/10.1159/000151696

Stoller, K. P. (2007). Hyperbaric oxygen and carbon monoxide poisoning: a critical review. Neurological Research, 29(2), 146-155. https://doi.org/10.1179/016164107X181770

Szalewska, D., Radkowski, M., Demkow, U., \& Winklewski, P. J. (2017). Exercise strategies to counteract brain aging effects. Advances in Experimental Medicine and Biology, 1020, 69-79. https://doi.org/10.1007/5584_2017_3

Tal, S., Hadanny, A., Berkovitz, N., Sasson, E., Ben-Jacob, E., \& Efrati, S. (2015). Hyperbaric oxygen may induce angiogenesis in patients suffering from prolonged post-concussion syndrome due to traumatic brain injury. Restorative Neurology and Neuroscience, 33(6), 943-951. https://doi.org/10.3233/RNN-150585

Tal, S., Hadanny, A., Sasson, E., Suzin, G., Efrati, S. (2017). Hyperbaric oxygen therapy can induce angiogenesis and regeneration of nerve fibers in traumatic brain injury patients. Frontiers in Human Neuroscience, 11. https://doi.org/10.3389/fnhum.2017.00508

Tapeantong, T., \& Poungvarin, N. (2009). Delayed encephalopathy and cognitive sequelae after acute carbon monoxide poisoning: Report of a case and review of the literature. Journal of the Medical Association of Thailand, 92(10), 1374-1379.

Thayer, J. F., \& Lane, R. D. (2000). A model of neurovisceral integration in emotion regulation and dysregulation. Journal of Affective Disorders, 61(3), 201-216. https://doi.org/10.1016/S0165-0327(00)00338-4

Thom, S. R., Bhopale, V. M., Fisher, D., Zhang, J., \& Gimotty, P. (2004). Delayed neuropathology after carbon monoxide poisoning is immune-mediated. Proceedings of the National Academy of Sciences of the United States of America, 101(37), 1366013665. https://doi.org/10.1073/pnas.0405642101

Thompson, L. W., Davis, G. C., Obrist, W. D., \& Heyman, A. (1976). Effects of hyperbaric oxygen on behavioral and physiological measures in elderly demented patients. Journals of Gerontology, 31(1), 23-28. https://doi.org/10.1093/geronj/31.1.23

Vila, J. F., Balcarce, P. E., Abiusi, G. R., Domínguez, R. O., \& Pisarello, J. B. (2005). Improvement in motor and cognitive impairment after hyperbaric oxygen therapy in a selected group of patients with cerebrovascular disease: A prospective singleblind controlled trial. Undersea and Hyperbaric Medicine, 32(5), 341-349.

Vonck, K., Raedt, R., Naulaerts, J., De Vogelaere, F., Thiery, E., Van Roost, D., Aldenkamp, B., Miatton, M., \& Boon, P. (2014). Vagus nerve stimulation..25 years later! What do we know about the effects on cognition? Neuroscience and Biobehavioral Reviews, 45, 63-71. https://doi.org/10.1016/j.neubiorev.2014.05.005

Walker, W. C., Franke, L. M., Cifu, D. X., \& Hart, B. B. (2014). Randomized, sham-controlled, feasibility trial of hyperbaric oxygen for service members with postconcussion syndrome: Cognitive and psychomotor outcomes 1 week postintervention. Neurorehabilitation and Neural Repair, 28(5), 420-432. https://doi.org/10.1177/1545968313516869

Walter, B., Daly, J., \& Bondurant, S. (1962). Effects of oxygen breathing on the heart rate, blood pressure, and cardiac index of normal men-resting, with reactive hyperemia, and after atropine oxygen breathing has been used extensively to treat a wide variety of clinical disorders. An underst. Journal of Clinical Investigation, 41(1), 126-132.

Wang, G. H., Zhang, X. G., Jiang, Z. L., Li, X., Peng, L. L., Li, Y. C., \& Wang, Y. (2010). Neuroprotective effects of hyperbaric oxygen treatment on traumatic brain injury in the rat. Journal of Neurotrauma, 27(9), 1733-1743. https://doi.org/10.1089/ neu.2009.1175

Wang, S., Tao, Z., Ding, S., Cheng, J., \& BS, Y., \& Wang, Y. (2009). Hyperbaric oxygen combined with donepezil in the treatment of vascular dementia. Chinese Journal of Physical Medicine and Rehabilitation, 31(7), 478-480.

Weaver, L. K. (2009). Clinical practice. Carbon monoxide poisoning. The New England Journal of Medicine, 360(12), 1217-1225. https://doi.org/10.1056/NEJMcp0808891

Weaver, L. K., Cifu, D., Hart, B., Wolf, G., \& Scott Miller, R. (2012). Hyperbaric oxygen for post-concussion syndrome: Design of Department of Defense clinical trials. Undersea and Hyperbaric Medicine, 39(4), 807-814.

Weaver, L. K., Cifu, D., Hart, B., Wolf, G., \& Scott Miller, R. (2013). Reply: Department of Defense trials for hyperbaric oxygen and TBI: Issues of study design and questionable conclusions. Undersea and Hyperbaric Medicine, 40(5), 471-472.

Weaver, L. K., Hopkins, R. O., Chan, K. J., Churchill, S., Elliott, C. G., Clemmer, T. P., Orme, J. F. J., Thomas, F. O., \& Morris, A. H. (2002). Hyperbaric oxygen for acute carbon monoxide poisoning. New England Journal of Medicine, 347(14), 1057-1067. https://doi.org/10.1056/NEJMoa013121 LK - http://rug.on. 
worldcat.org/atoztitles/link/?sid=EMBASE \&issn $=00284793 \&$ $\mathrm{id}=$ doi: $10.1056 \% 2 \mathrm{FNEJMoa} 013121 \&$ atitle $=$ Hyperbaric + oxygen + for + acute + carbon + monoxide + poisoning $\&$ stitle $=$ New +Engl.+J.+Med. \& title $=$ New + England + Journal+ of + Medicine $\&$ volume $=347 \&$ issue $=14 \&$ spage $=1057 \&$ epage $=$ $1067 \&$ aulast $=$ Weave $r \&$ aufir $s t=$ Linde $11+K$. \& auinit $=$ L.K.\&aufull $=$ Weaver + L.K.\&coden $=$ NEJMA $\&$ isbn $=\&$ pages $=$ $1057-1067 \&$ date $=2002 \&$ auinit $1=$ L\&auinitm $=K$

Weaver, L. K., Valentine, K. J., \& Hopkins, R. O. (2007). Carbon monoxide poisoning: Risk factors for cognitive sequelae and the role of hyperbaric oxygen. American Journal of Respiratory and Critical Care Medicine, 176(5), 491-497. https://doi. org/10.1164/rccm.200701-026OC

Weaver, L. K., Wilson, S. H., Lindblad, A. S., Churchill, S., Deru, K., Price, R. C., Williams, C. S., Orrison, W. W., Walker, J. M., Meehan, A., \& Mirow, S. (2018). Hyperbaric oxygen for postconcussive symptoms in United States military service members: A randomized clinical trial. Undersea and Hyperbaric Medicine, 45(2), 129-156. https://doi.org/10.22462/03.04.2018.1

Winklewski, P. J., Kot, J., Frydrychowski, A. F., Nuckowska, M. K., \& Tkachenko, Y. (2013). Effects of diving and oxygen on autonomic nervous system and cerebral blood flow. Diving and Hyperbaric Medicine, 43(3), 148-156.

Winklewski, P. J., Sabisz, A., Naumczyk, P., Jodzio, K., Szurowska, E., \& Szarmach, A. (2018). Understanding the physiopathology behind axial and radial diffusivity changes-what do we Know? In Frontiers in Neurology (Vol. 9, Issue FEB). Frontiers Media S.A. https://doi.org/10.3389/fneur.2018.00092

Wolf, E. G., Baugh, L. M., Schubert Kabban, C. M., Richards, M. F., \& Prye, J. (2015). Cognitive function in a traumatic brain injury hyperbaric oxygen randomized trial. Undersea and Hyperbaric Medicine, 42(4), 313-332.

Wolf, G., Cifu, D., Baugh, L., Carne, W., \& Profenna, L. (2012). The effect of hyperbaric oxygen on symptoms after mild traumatic brain injury. Journal of Neurotrauma, 29(17), 2606-2612. https://doi.org/10.1089/neu.2012.2549

Wright, J. K., Zant, E., Groom, K., Schlegel, R. E., \& Gilliland, K. (2009). Case report: Treatment of mild traumatic brain injury with hyperbaric oxygen. Undersea \& Hyperbaric Medicine: Journal of the Undersea and Hyperbaric Medical Society, Inc, 36(6), 391-399. http://www.ncbi.nlm.nih.gov/pubmed/20112530

Xiang, W., Xue, H., Wang, B., Li, Y., Zhang, J., Jiang, C., et al. (2017a). Combined application of dexamethasone and hyperbaric oxygen therapy yields better efficacy for patients with delayed encephalopathy after acute carbon monoxide poisoning. Drug Design, Development and Therapy, 11, 513-519. https://doi. org/10.2147/DDDT.S126569.

Xiang, W., Xue, H., Wang, B., Li, Y., Zhang, J., Jiang, C., \& Pang, J. (2017b). Efficacy of N-Butylphthalide and Hyperbaric Oxygen Therapy on Cognitive Dysfunction in Patients with Delayed Encephalopathy After Acute Carbon Monoxide Poisoning. In Medical science monitor (Vol. 23, pp. 1501-1506). http:// onlinelibrary.wiley.com/o/cochrane/clcentral/articles/219/ CN-01368219/frame.html

Xu, Y., Wang, Q., Qu, Z., Yang, J., Zhang, X., \& Zhao, Y. (2019). Protective Effect of Hyperbaric Oxygen Therapy on Cognitive Function in Patients with Vascular Dementia. Cell Transplantation, 28(8), 1071-1075. https://doi.org/10.1177/0963689719853540

Yeh, Z. T., Tsai, C. F., Yip, P. K., Lo, C. Y., Peng, S. M., Chen, S. Y., \& Kung, L. Y. (2014). Neuropsychological performance in patients with carbon monoxide poisoning. Applied Neuropsychology:Adult, 21(4), 278-287. https://doi.org/10.1080/23279095.2013.811670

You, Q., Li, L., Xiong, S. Q., Yan, Y. F., Li, D., Yan, N. N., Chen, H. P., \& Liu, Y. P. (2019). Meta-analysis on the efficacy and safety of hyperbaric oxygen as adjunctive therapy for vascular dementia. In Frontiers in Aging Neuroscience (Vol. 11, Issue APR). Frontiers Media S.A. https://doi.org/10.3389/fnagi.2019.00086

Zádori, A., Ágoston, V. A., Demeter, K., Hádinger, N., Várady, L., Kohídi, T., Gobl, A., Nagy, Z., \& Madarász, E. (2011). Survival and differentiation of neuroectodermal cells with stem cell properties at different oxygen levels. Experimental Neurology, 227(1), 136-148. https://doi.org/10.1016/j.expneurol.2010.10.004

Zagami, A. S., Lethlean, A. K., \& Mellick, R. (1993). Delayed neurological deterioration following carbon monoxide poisoning: MRI findings. Journal of Neurology, 240(2), 113-116. https:// doi.org/10.1007/bf00858727

Zasler, N. D., Katz, D. I., \& Zafonte, R. D. (2007). Brain Injury Medicine : Principles and Practice. Demos.

Zhang, J., Guo, Y., Li, W., Li, G., \& Chen, Y. (2020). The efficacy of N-butylphthalide and dexamethasone combined with hyperbaric oxygen on delayed encephalopathy after acute carbon monoxide poisoning. Drug Design, Development and Therapy, 14, 13331339. https://doi.org/10.2147/DDDT.S217010

Zhang, T., Yang, Q. W., Wang, S. N., Wang, J. Z., Wang, Q., Wang, Y., \& Luo, Y. J. (2010). Hyperbaric oxygen therapy improves neurogenesis and brain blood supply in piriform cortex in rats with vascular dementia. Brain Injury, 24(11), 1350-1357. https://doi. org/10.3109/02699052.2010.504525

Zhang, Y. P., Cai, J., Shields, L. B. E., Liu, N., Xu, X. M., \& Shields, C. B. (2014). Traumatic Brain Injury Using Mouse Models. In Translational Stroke Research (Vol. 5, Issue 4, pp. 454-471). Springer US. https://doi.org/10.1007/s12975-014-0327-0

Zhao, B., Pan, Y., Wang, Z., Xu, H., \& Song, X. (2017). Hyperbaric oxygen pretreatment improves cognition and reduces hippocampal damage via p38 mitogen-activated protein kinase in a rat model. Yonsei Medical Journal, 58(1), 131-138. https://doi.org/ 10.3349/ymj.2017.58.1.131

Zhou, Z., Daugherty, W. P., Sun, D., Levasseur, J. E., Altememi, N., Hamm, R. J., Rockswold, G. L., \& Bullock, M. R. (2007). Protection of mitochondrial function and improvement in cognitive recovery in rats treated with hyperbaric oxygen following lateral fluid-percussion injury. Journal of Neurosurgery, 106(4), 687-694. https://doi.org/10.3171/jns.2007.106.4.687

Publisher's Note Springer Nature remains neutral with regard to jurisdictional claims in published maps and institutional affiliations. 